\title{
New views of the distant stellar halo
}

\author{
Robyn E. Sanderson, ${ }^{1,2 \star} \dagger$ Amy Secunda, ${ }^{1}$ Kathryn V. Johnston ${ }^{1}$ \\ and John J. Bochanski ${ }^{3}$ \\ ${ }^{1}$ Department of Astronomy, Columbia University, 550 W 120th St, New York, NY 10027, USA \\ ${ }^{2}$ TAPIR, Caltech, MC 301-17, 1200 E. California Blvd., Pasadena, CA 91125, USA \\ ${ }^{3}$ Department of Chemistry, Biochemistry and Physics, Rider University, 2083 Lawrenceville Road, Lawrenceville, NJ 08648, USA
}

Accepted 2017 June 22. Received 2017 June 22; in original form 2016 September 20

\begin{abstract}
Currently, only a small number of Milky Way (MW) stars are known to exist beyond $100 \mathrm{kpc}$ from the Galactic Centre. Though the distribution of these stars in the outer halo is believed to be sparse, they can provide evidence of more recent accretion events than in the inner halo and help map out the MW's dark matter halo to its virial radius. We have re-examined the outermost regions of 11 existing stellar halo models with two synthetic surveys: one mimicking present-day searches for distant M giants and another mimicking RR Lyra (RRL) projections for the Large Synoptic Survey Telescope (LSST). Our models suggest that colour and proper motion cuts currently used to select $\mathrm{M}$ giant candidates for follow-up successfully remove nearly all self-contamination from foreground halo dwarf stars and are useful for focusing observations on distant $\mathrm{M}$ giants, of which there are thousands to tens of thousands beyond $100 \mathrm{kpc}$ in our models. We likewise expect that LSST will identify comparable numbers of RRLe at these distances. We demonstrate that several observable properties of both tracers, such as proximity of neighbouring stars, proper motions and distances (for RRLe), could help us separate different accreted dwarf galaxies from one another in the distant MW halo. We also discuss prospects for using ratios of M giants to RRLe as a proxy for accretion time, which in the future could provide new constraints on the recent accretion history of our Galaxy.
\end{abstract}

Key words: stars: variables: RR Lyrae - Galaxy: formation - Galaxy: halo - Galaxy: kinematics and dynamics-Galaxy: stellar content-Galaxy: structure.

\section{INTRODUCTION}

Stellar haloes of spiral galaxies typically contain of the order of a few per cent of the total number of stars associated with their host dark matter haloes, spread over spatial scales 10 times larger than the discs that they surround. Hence, they are insignificant in terms of understanding the bulk of baryonic material that has occurred throughout the history of the Universe, and their extremely low densities (and corresponding surface brightness) make them in any case difficult to study. However, two properties of stellar haloes make them uniquely interesting. First, it is here that it is most productive to search for stars that were not formed in the current host halo, but rather accreted from other objects. Hence, the properties of the stellar populations of the halo can tell us something both about the accretion histories of galaxies and the properties of the (now-dead) dwarf galaxies that formed them. Secondly, the low total mass and vast spatial scales that halo stars explore make them

^E-mail: robyn@ caltech.edu

$\dagger$ NSF Astronomy and Astrophysics Postdoctoral Fellow. powerful probes of the mass and structure of dark matter haloes that surround all galaxies.

The production of vast catalogues of faint stars around our own (see Ivezić, Beers \& Jurić 2012, for a review) and other (Ferguson et al. 2002) galaxies have for the first time allowed the global structure of several stellar haloes to be convincingly mapped (Ibata et al. 2014). These studies have also revealed the presence of a significant contribution of substructure in space (Ferguson et al. 2002; Newberg et al. 2002; Belokurov et al. 2006a) and velocity (Gilbert et al. 2009; Schlaufman et al. 2009), which can be attributed to the hierarchical nature of galaxy formation (Bullock, Kravtsov \& Weinberg 2001) - the substructures are the debris from the destruction of infalling dwarf galaxies. Comparisons with concurrent theoretical work suggest broad consistency of these observations with the expectations for the scales, structure and frequency of substructure in stellar haloes built within the $\Lambda$ cold dark matter $(\Lambda \mathrm{CDM})$ paradigm (Bell et al. 2008, 2010; Xue et al. 2011). Some of these substructures have been exploited as probes of the underlying gravitational potential (Helmi 2004; Johnston, Law \& Majewski 2005; Law, Johnston \& Majewski 2005; Law, Majewski \& Johnston 2009; Willett et al. 2009; Koposov, Rix \& Hogg 2010; 
Law \& Majewski 2010; Newberg et al. 2010; Lux et al. 2012; Sesar et al. 2013; Vera-Ciro \& Helmi 2013; Küpper et al. 2015; Pearson et al. 2015).

A significant observational challenge in this field is the mapping and interpretation of the outermost regions of galactic haloes all the way out to the virial radius $(\sim 300 \mathrm{kpc}$ for a Milky Way-mass galaxy). M31 is the only galaxy in the Universe for which a global map has been made on these scales, reaching to $\sim 150 \mathrm{kpc}$ (Ibata et al. 2014). In contrast, for the Milky Way (MW), the views of the stellar halo afforded by main-sequence turnoff (MSTO) stars selected from the Sloan Digital Sky Survey (SDSS) extend to $\sim 40 \mathrm{kpc}$ and the M giants extracted from 2MASS reach distances of less than $100 \mathrm{kpc}$ (Majewski et al. 2003; Belokurov et al. 2006a). In the next few years, data releases from the Gaia satellite promise to fill in these maps with vast numbers of stars and additional dimensions of information, but Gaia's magnitude limit of roughly $V \sim 20$ again restricts sensitivity to within roughly $100 \mathrm{kpc}$ of the Galactic Centre for bright giant tracers.

The number of MW stars known to lie beyond $100 \mathrm{kpc}$ from the Galactic Centre is still very small, but steadily growing. Largearea surveys with deep, precise photometry have been critically important to identifying relatively rare, but luminous, halo stars. Two classes of stars are bright enough to be observed beyond $120 \mathrm{kpc}$ with current surveys: blue horizontal branch (BHB) stars and M giants. Deason et al. (2012) selected a sample of seven spectroscopically confirmed BHB stars in SDSS with distances of $80 \mathrm{kpc}<d<150 \mathrm{kpc}$. At distances greater than $150 \mathrm{kpc}$, only $\mathrm{M}$ giants are bright enough to be readily observed in modern day surveys. Bochanski et al. (2014a) assembled a sample of nearly $500 \mathrm{M}$ giant candidate stars with optical and infrared photometry from SDSS and the UKIRT Infrared Deep Sky Survey (UKIDSS). The $\mathrm{M}$ giants in the Bochanski et al. sample can be seen from 30 to $\sim 300 \mathrm{kpc}$, making them the first to probe the stellar content of the MW near the virial radius. Unfortunately, photometry and the lack of proper motions (PMs) are not sufficient to confirm that a star is an $\mathrm{M}$ giant, making spectroscopy necessary. Despite an estimated contamination rate near 80 per cent, Bochanski et al. (2014b) have already spectroscopically confirmed two M giants with estimated distances over $200 \mathrm{kpc}$. The most distant M giant known, ULAS J001535.72+015549.6, has a distance of $274 \pm 74 \mathrm{kpc}$. In total, the UKIDSS sample has yielded 10 confirmed M giants to date, most being part of the Sagittarius dwarf galaxy remnant.

Further into the future, we can anticipate dramatic additions to these outer halo detections as the Large Synoptic Survey Telescope (LSST; Ivezic et al. 2008) produces catalogues of stars as faint as $g=24.5$ in a single pointing, corresponding to a distance limit of $\sim 50 \mathrm{kpc}$ for an MSTO star and $\sim 600 \mathrm{kpc}$ for an RR Lyra (RRL) star. After 5 years, the co-added data will reach $g=27$, out to $\sim 300 \mathrm{kpc}$ for an MSTO and $\sim 3 \mathrm{Mpc}$ for an RRL. It is as yet unclear what to expect in this regime. Model stellar haloes show them becoming more dominated by substructure at larger galactocentric radii as the dynamical time-scales become comparable to the age of the Universe and the debris from the few recent accretion events has little time to phase-mix away (Johnston et al. 2008). M31's stellar halo extends at least to $150 \mathrm{kpc}$ and is richly substructured (Ibata et al. 2014), but the stochastic nature of hierarchical structure formation ensures a vast variety in stellar halo structures, especially on these spatial scales, so the MW's stellar halo could differ dramatically.

Even if the populations of stars in the outermost halo prove to be very sparse, they will have some important implications: they will provide a view of accretion (or perhaps lack of accretion?) in a new and unique regime, likely more sensitive recent events; and they will provide dynamical tracers to map the dark matter halo all the way out to the virial radius. This paper is motivated by the steadily growing number of stars known to be beyond $100 \mathrm{kpc}$ from the Galactic Centre, as well as the longer term prospects for LSST, to re-examine the outermost reaches of 11 existing stellar halo models (Bullock \& Johnston 2005) in order to explore our expectations for these populations in a little more detail. In particular, this study looks at two different types of stars that might be selected in current and future stellar catalogues: colour-selected $\mathrm{M}$ giants and timedomain-selected RRLe. For each tracer, we examine the trends and diversity in numbers of stars and properties of objects from which they came in the models. We also discuss the likelihood of being able to make associations between stars from their observed properties associations that will increase our ability to reconstruct both the full accretion history of our Galaxy and the structure of its dark matter halo.

This paper is organized as follows: in Section 2, we describe the computational tools (the mock haloes and GALAXIA) used to generate synthetic stellar populations in the outer halo; in Section 3, we describe how we generated mock surveys for two tracer populations, $M$ giants (Section 3.1) and RRLe (Section 3.2), and compare the view of the stellar halo obtained for each tracer (Section 3.3-3.5). In Section 4, we discuss prospects for finding building blocks by searching for nearby pairs of stars from the same tracer population. In Section 5, we consider what information is most useful to distinguish stars from different accreted building blocks. In Section 6, we consider the expectations of our models for the eventual results of the search for distant $\mathrm{M}$ giants from UKIDSS, as described in Bochanski et al. (2014a). In Section 7, we discuss the possibility of using ratios of the two tracer populations to reconstruct the MW's accretion history. In Section 8, we summarize our findings, draw some conclusions and indicate some directions for future work.

\section{TOOLBOX}

We use the set of publicly available ${ }^{1}$ mock stellar haloes from Bullock \& Johnston (2005), and look at M giant and RRL stellar tracers by generating synthetic surveys with GALAXIA ${ }^{2}$ (Sharma et al. 2011b). In this section, we describe the relevant features of these two tools for our purposes.

\subsection{Mock stellar halo simulations}

The stellar halo models described in Bullock \& Johnston (2005) were built entirely from accretion events drawn from histories representing random realizations of the formation of a MW-type galaxy in a $\Lambda$ CDM universe. The phase-space structure in the models was constructed by superposing the final positions and velocities of particles at the end point of individual $N$-body simulations of dwarf galaxies disrupting around a parent galaxy matching the cosmological model accretion event history. All subhaloes crossing within the virial radius of the parent galaxy ( $282 \mathrm{kpc}$ at present day) were tracked. In this work, we will refer to these tracked subhaloes, and the stellar distributions associated with them, as the 'building blocks' of the stellar halo. The 100000 massive particles in each of these simulated objects had equal dark matter masses and were

\footnotetext{
${ }^{1}$ http://user.astro.columbia.edu/ kvj/halos/

${ }^{2}$ http://galaxia.sourceforge.net
} 
given (varying) associated mass-to-light ratios in such a way that the luminous material reproduced the structural scaling relations observed for Local Group dwarf galaxies. The level of resolution of the phase-space structure of resulting stellar halo was increased by introducing an additional 100000 massless test particles with the same energy distribution as the 20 per cent most tightly bound dark matter particles.

In order to model the stellar populations, the dark matter growth of each satellite prior to accretion was approximated (for a given satellite mass and concentration) by the smooth fitting function of Wechsler et al. (2002). The associated gas accretion was represented using a formalism to mimic the satellite-mass-dependent effect of ionizing background radiation (see Bullock \& Johnston for full details). The corresponding development of stellar populations within each dwarf was followed using a simple leaky-accreting-box model of star formation and chemical enrichment, with the star formation rate assumed to be proportional to the gas accretion rate and abruptly truncated upon accretion on to the MW (Robertson et al. 2005; Font et al. 2006). The parameters of the enrichment model were fixed so as to reproduce the mass-metallicity relation of observed dwarfs in the Local Group. The model produced a range of stellar populations in each satellite with metallicity decreasing monotonically with age.

Two key attributes of the Bullock \& Johnston (2005) models should be borne in mind throughout the rest of this paper. First, the models only represent the portion of halo stars that were formed in and subsequently accreted from other dark matter haloes. Fully selfconsistent, cosmological hydrodynamical simulations of the formation of MW-type galaxies have typically been found to contain an additional population beyond their galactic discs that formed within the main galaxy's own dark matter halo (Abadi, Navarro \& Steinmetz 2006; Zolotov et al. 2009; Font et al. 2011; Tissera et al. 2013; Pillepich, Madau \& Mayer 2015). However, while these models typically differ on the percentage and radial distribution of stellar halo stars formed this way, none predict a significant portion beyond $\sim 50 \mathrm{kpc}$ from the Galactic Centre. Hence, we anticipate our consideration only of accreted populations to be a valid simplification for the study of the outer halo.

Secondly, the models were built to match the number and luminosities of the dozen contemporaneously known satellite galaxies of the MW, and hence do not contain the more numerous population of less luminous ultra-faint dwarf (UFD) satellite galaxies that have been discovered since that time (e.g. Willman et al. 2005; Belokurov et al. 2006b; Drlica-Wagner et al. 2015), nor stars from the ancestral analogues of the surviving UFDs that would have been disrupted at earlier times. However, we would expect these many smaller galaxies to contribute stars that might occupy unique corners of stellar populations and abundance space. In particular, these galaxies would be too metal poor to have any $\mathrm{M}$ giants associated with them, so their absence in the models does not affect our comparison with UKIDSS. On the other hand, their contributions could alter the size and phase-space structure of the RRL halo.

Given these limitations, our examination of these models is intended as indicative, rather than predictive. This would be true for any stellar halo models that could be examined. While there is very broad agreement among the different models (and across techniques) that stars in the halo beyond $50 \mathrm{kpc}$ are most likely accreted, there is no clear consensus on the amount or type of material or expected phase-space structure (Bullock \& Johnston 2005; De Lucia \& Helmi 2008; Cooper et al. 2010; Helmi et al. 2011). Part of this apparent disagreement is undoubtedly due to the stochastic nature of hierarchical structure formation (as reflected in this paper in Figs 6 and 7). However, some can be attributed to differences between representations of either the physics or objects in the Universe in the models themselves. For one example, see discussion in Bailin et al. (2014) on how the presence or absence of a disc potential can change stellar halo properties in models. These differences have been driven by computational rather than physical considerations models with sufficiently self-consistent representations of the range of physical processes needed to be predictive and with high enough resolution to compare to star-count studies do not yet exist in large numbers, although they are on the horizon (Wetzel et al. 2016).

\subsection{Synthetic surveys with GALAXIA}

To generate synthetic surveys of the mock stellar haloes, we use the public software package GALAXIA (Sharma et al. 2011b). This code resamples the $N$-body particle representations of the building blocks of a given mock halo from Bullock \& Johnston, following the luminosity weights assigned to each $N$-body particle as discussed in Section 2.1 and presuming stellar populations represented by the Padova isochrones (Bertelli et al. 1994; Marigo et al. 2008).

For each $N$-body particle in one of the mock haloes, GALAXIA defines an isochrone corresponding to the age and metallicity of the particle that was assigned by the star formation model for that accreted dwarf galaxy. This is accomplished by interpolating between tabulated isochrones for a range of ages and metallicities. The code then calculates the number of stars that, when sampled from this isochrone, add up to the stellar luminosity of the $N$-body particle. It determines how many of these stars fall inside the colourmagnitude box, sky area and distance limits specified by the user, and generates a list of the synthetic stars to be included in the survey. Each synthetic star is assigned a three-dimensional position and velocity in Cartesian heliocentric coordinates sampled from the $\mathrm{N}$-body distribution, absolute magnitudes in each of the specified filters, age, metallicity, and alpha abundance from the parent particle, and tags identifying (1) the accreted building block to which that star belongs, and (2) whether or not that building block is still gravitationally self-bound at the present day. Apparent magnitudes and conversions to observers' coordinates for position and velocity [sky positions, heliocentric distances, PMs, radial velocities (RVs)] can be computed by accompanying scripts run on the completed synthetic survey. The solar position and velocity used by GALAXIA are also reported so that the heliocentric Cartesian coordinates can be converted to the galactocentric frame self-consistently.

Stellar streams are extremely cold structures compared to the parent halo, so each individual building block in a given halo is resampled individually. The code performs the phase-space resampling in two 3D subspaces (positions and velocities) rather than in the full $6 \mathrm{D}$ space, and so considering each building block individually ensures that the sampler does not overestimate their configurationspace thickness or velocity dispersion through confusion between streams from multiple building blocks. We refer the interested reader to Sharma et al. (2011b) for more details of the resampling process.

\section{SYNTHETIC SURVEYS OF M GIANTS AND RRLE}

In this work, we consider synthetic surveys of the 11 mock haloes for two different tracer populations: $M$ giants and RRLe.

$\mathrm{M}$ giant stars are an interesting tracer both because their intrinsic luminosity allows us to use them to trace the outermost reaches of our Galaxy and because they can be efficiently separated from foreground dwarf stars using a combination of colour-colour selections. Searches are ongoing for distant $\mathrm{M}$ giants in wide-field surveys, and 
Table 1. Stellar tracers used in this work.

\begin{tabular}{lccccc}
\hline Population & Abs. mag & App. mag range & Colour range & Approx. distance range (kpc) & Note \\
\hline RRLe & $-1.0<M_{K_{s}}<0.1$ & $10<m_{K_{s}}<24.5$ & $0.13<J-K_{s}<0.363$ & $d<600$ & as in Sharma et al. (2011a) \\
M giants & $0.05<M_{J}<0.25$ & $12.5<J<18.5$ & $0.05<H-K<0.4$ & $30<d<300$ & as in Bochanski et al. (2014b) \\
\hline
\end{tabular}

have already yielded the two most distant known stars in the MW (Bochanski et al. 2014b). More $\mathrm{M}$ giants tend to form in galaxies with relatively high metallicity $([\mathrm{Fe} / \mathrm{H}] \gtrsim 1)$ and thus trace the relatively more massive accreted objects in the stellar halo. Although these tracers are fairly numerous, the best prospects for distance estimates are probably around 10-20 per cent relative distance error, and current known distant $\mathrm{M}$ giants have poorly constrained distances ( $\sim 50-100$ per cent error).

RRLe are less luminous and less numerous than $M$ giants but have several distinct advantages of their own. They are fairly unambiguous to identify from their light curves if multiple epochs of observations are available, so no confusion is expected with foreground objects. RRLe also follow a period-luminosity relation that can be used to obtain very accurate distances: to around 5 per cent in the optical (Clementini et al. 2003; Sesar et al. 2010) and less than 2 per cent in the IR (Beaton et al. 2016). Finally, they explore different types of objects than $\mathrm{M}$ giant stars since they are only present in populations with $[\mathrm{Fe} / \mathrm{H}] \lesssim 1$. This makes them powerful tracers of the MW accretion history that are complementary to $\mathrm{M}$ giants. Their very accurate distances can both help to untangle different accreted satellite galaxies (in particular, the smaller dwarf galaxies) and better constrain the mass distribution.

In this section, we describe how the two sets of synthetic surveys were generated, and give an overview and brief comparison of their properties.

\subsection{A synthetic survey of distant $M$ giants}

Our synthetic M giant surveys are modelled after the depth and selection function of UKIDSS used to identify possible M giants for spectroscopic follow-up, as described in Bochanski et al. (2014a). We used GALAXIA to generate synthetic stars falling into a box in colour-magnitude space containing $\mathbf{M}$ giants, convolved these synthetic stars with photometric and PM errors corresponding to present and future data sets, and selected distant $\mathrm{M}$ giants from the resulting mock catalogue with the same infrared and visible colour cuts as the candidate sample in Bochanski et al. (2014a), for each of the 11 different mock stellar haloes in Bullock \& Johnston (2005). Here we describe the steps in this process in detail.

\subsubsection{Generating the synthetic, error-convolved survey}

We use GalaXia (Sharma et al. 2011b) to construct a synthetic all-sky survey of the 11 mock stellar haloes in Bullock \& Johnston (2005). For this initial effort, we do not include foregrounds from the Galactic disc and any smooth halo component, focusing only on the accreted halo. This is somewhat justifiable since we are examining a sample at 100-300 kpc in the halo, but it fails to account for the contamination of the sample by dwarf stars in the disc (Bochanski et al. estimate this significant contamination to be roughly 80 per cent). We restrict the synthetic survey generated by GALAXIA to the region of colour-magnitude space listed in Table 1, which is the starting point for Bochanski et al.'s selection of a sample of likely $\mathrm{M}$ giants from UKIDSS. We generate UBV $J, H$ and $K$ infrared magnitudes and Sloan $g$ and $i$ visual magnitudes for all stars in this
Table 2. Constants for photometric error models for 'present-day' survey (UKIDSS + SDSS).

\begin{tabular}{lclc}
\hline Band & $a$ & \multicolumn{1}{c}{$b$} & $c$ \\
\hline Sloan $g$ & $7.5 \times 10^{-7}$ & 0.553 & -0.0361 \\
Sloan $i$ & $2.1 \times 10^{-9}$ & 0.814 & 0.0134 \\
UBV $J$ & $8.7 \times 10^{-10}$ & 0.9993 & $1.74 \times 10^{-4}$ \\
UBV $H$ & $1.6 \times 10^{-8}$ & 0.870 & $1.62 \times 10^{-5}$ \\
UBV $K$ & $2.0 \times 10^{-8}$ & 0.888 & $-2.65 \times 10^{-4}$ \\
\hline
\end{tabular}

colour-magnitude box, as well as sky positions, parallaxes, RVs and PMs calculated from the six-dimensional Cartesian, galactocentric phase-space coordinates returned by GALAXIA.

We then convolve ${ }^{3}$ the photometry and PMs (which are used together to select the M giants) with error models based on presentday surveys. We presume UKIDSS-like photometric errors for the $J, H$ and $K$ bands and Sloan photometric errors for $g$ and $i$, with magnitude dependence $\sigma_{m}$ in all bands defined by the function

$\sigma_{m}=a \mathrm{e}^{b m}+c$,

where $m$ is the magnitude, and $a, b$ and $c$ are constants that are different in each band, given in Table 2. We presume a constant PM error of $2 \mathrm{mas}^{-1} \mathrm{yr}$ based on the SDSS/USNO-B $50 \mathrm{yr}$ baseline (Sloan DR10).

\subsubsection{Selecting candidate $M$ giants}

We mimic the series of cuts described in Bochanski et al. (2014a) on our synthetic survey, including photometric errors, to select $\mathrm{M}$ giants from the synthetic all-sky surveys. These cuts use colour information to reduce the number of foreground $M$ dwarfs and background quasars. Starting with roughly 10 million stars from each of the 11 mock haloes that fall within the broad colour and magnitude ranges in Table 1, we first choose objects inside the box in IR colour-colour space shown in Fig. 1 , to conservatively separate giants from dwarf stars. The left-hand panel of Fig. 1 shows the selection operating on the unconvolved synthetic survey, while the right-hand panel shows the selection on the error-convolved data. As shown on the left, this cut would indeed select only giants given perfect data, but as shown on the right, the photometric errors also scatter dwarf stars into the box even though the only contamination here is from other stars in the accreted halo. Some evidence of this contamination is seen in the broadening of the black contours indicating the selection in the right-hand panel, relative to the red contours indicating the giants only. Many giants also lie outside the selection box in both the convolved and unconvolved samples.

The effect of each successive selection described in this section is illustrated in Fig. 3 in terms of the distribution of number of dwarf and giant stars remaining (left-hand panel) and number of stars

\footnotetext{
${ }^{3}$ For each star in each coordinate, we calculate the error $\sigma$ from a model based on the 'true' (unconvolved) values generated by the synthetic survey, and then draw a random sample from a Gaussian with width $\sigma$ centred on the 'true' value.
} 

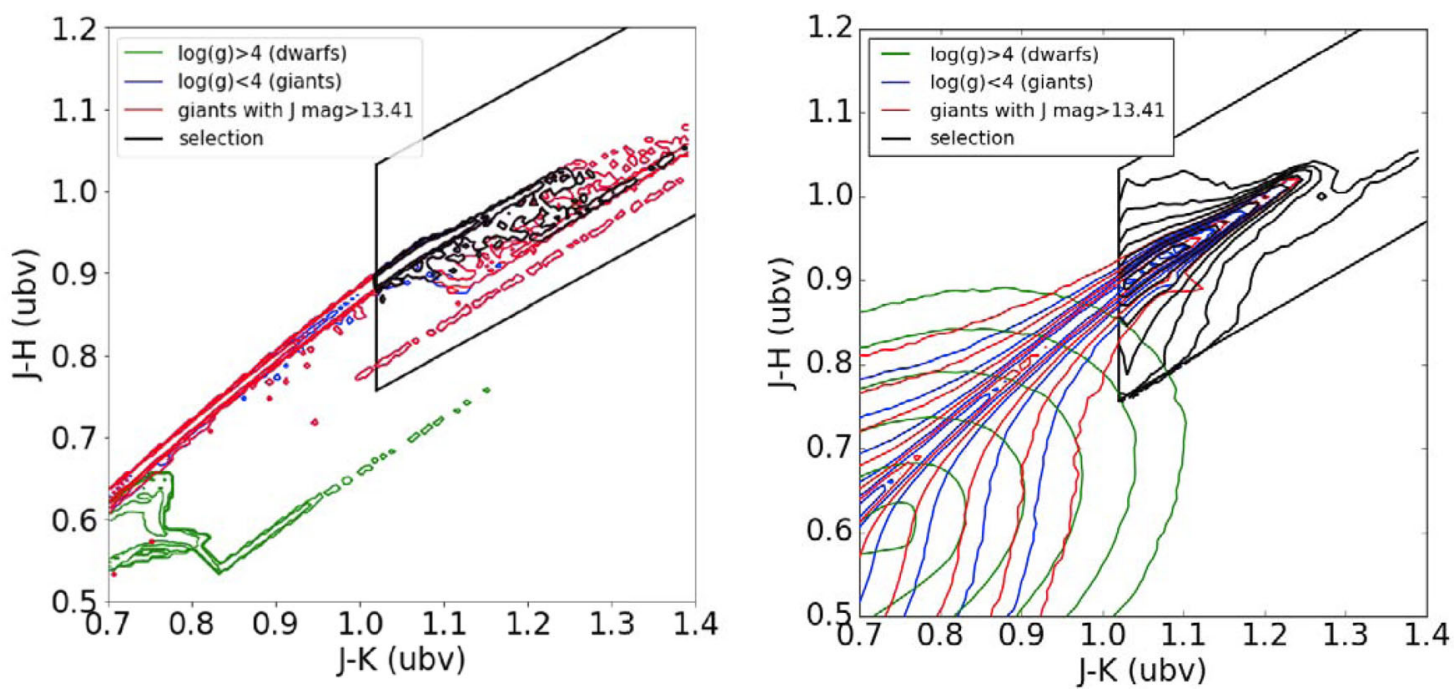

Figure 1. Example of selection of giant stars in infrared colour-colour space for one of the 11 haloes studied (see Section 3.1.2). In both panels, stars inside the black box are selected. Green contours show synthetic stars with $\log g>4$ (dwarf stars), blue contours are synthetic stars with $\log g<4$ (giants), red indicates giants with $J$ magnitudes greater than 13.41 and black contours show stars selected based on the criteria outlined in Section 3.1.2. Left: the selection operating on the synthetic survey without error convolution. Right: the same selection applied to the error-convolved synthetic survey. The selected population shown in black includes the colour-colour cut shown in Fig. 2.
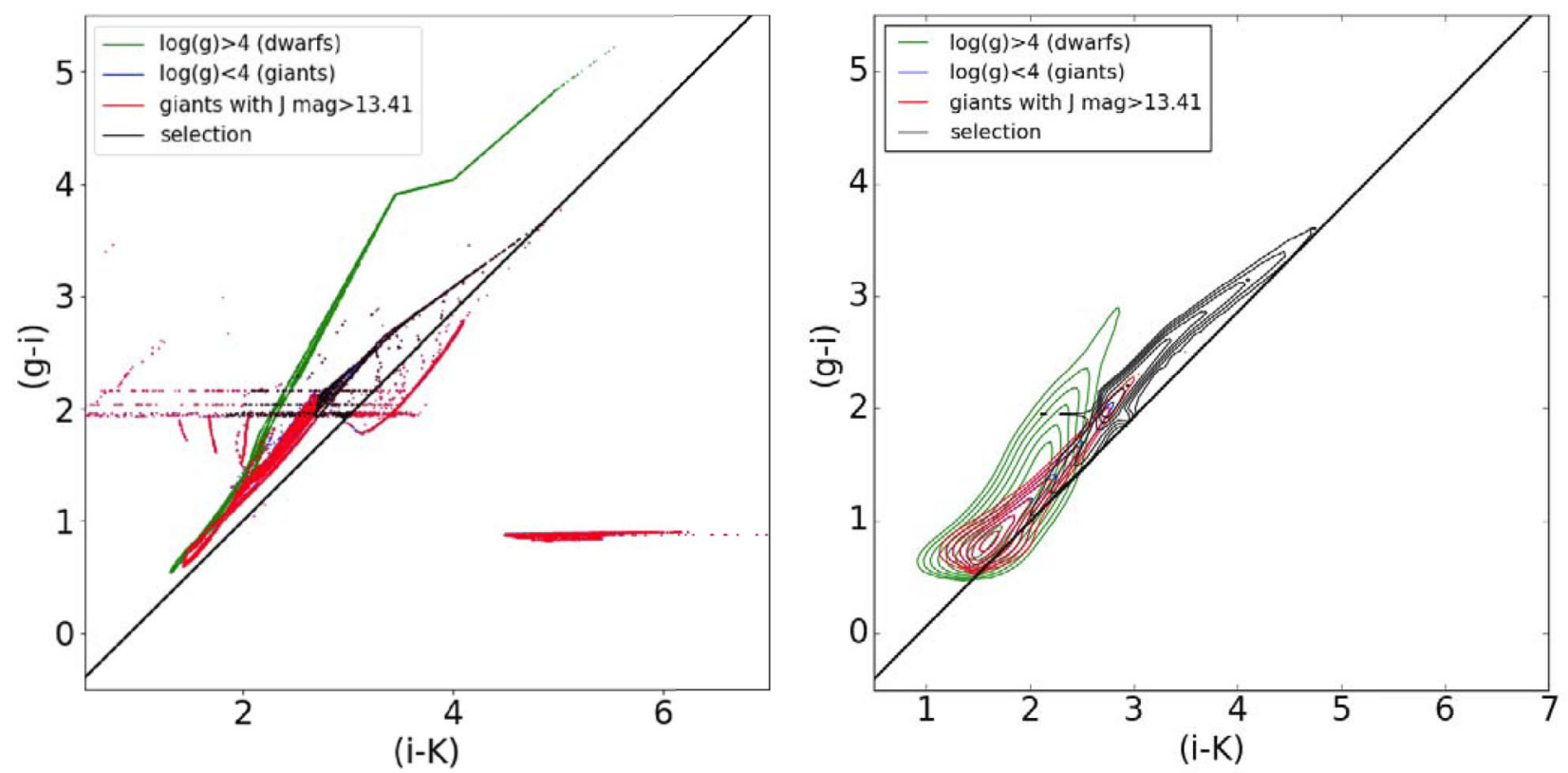

Figure 2. Selection in colour-colour space to eliminate quasars, shown for the same halo as Fig. 1 (see Section 3.1.2). Stars above the diagonal line are selected. Colour scheme is as in Fig. 1. Left: the selection operating on the synthetic survey without error convolution. Right: the same selection applied to the error-convolved synthetic survey. The selected population shown in black includes the colour-colour cut shown in Fig. 1.

remaining as a function of true distance (right-hand panel). The left-hand panel of Fig. 3 shows that with this first colour-colour cut, the number of giant stars is reduced even more than the number of foreground halo dwarf stars, which would seem to run counter to the intention of this selection. However, in reality, the discarded giants would be extremely difficult to separate from foreground dwarf stars in the disc, which are vastly more numerous than the halo dwarf stars considered here. The right-hand panel of Fig. 3 shows the distance distribution of the selected stars (red line) for one of the 11 haloes.

Another selection is then made in IR-optical space to separate stars from quasars, as shown in Fig. 2. Stars below the black line (i.e. in the lower-right corner of the plot) are discarded. As before, this figure compares the selection made on the unconvolved and error-convolved synthetic surveys in the left- and right-hand panels, respectively. There are no quasars in our synthetic survey, but as seen on the left, this cut does still eliminate some real giants as shown in the figure, even when photometric errors are not taken into account. However, the left-hand panel of Fig. 3 shows that this cut (the green line) has only a small effect on the target population at any distance, and is not very distance dependent.

Comparing the numbers of $\mathrm{M}$ giants selected by our colour cuts made on the unconvolved data versus the same cuts made on errorconvolved data, we find that convolved data lead to a scattering in 

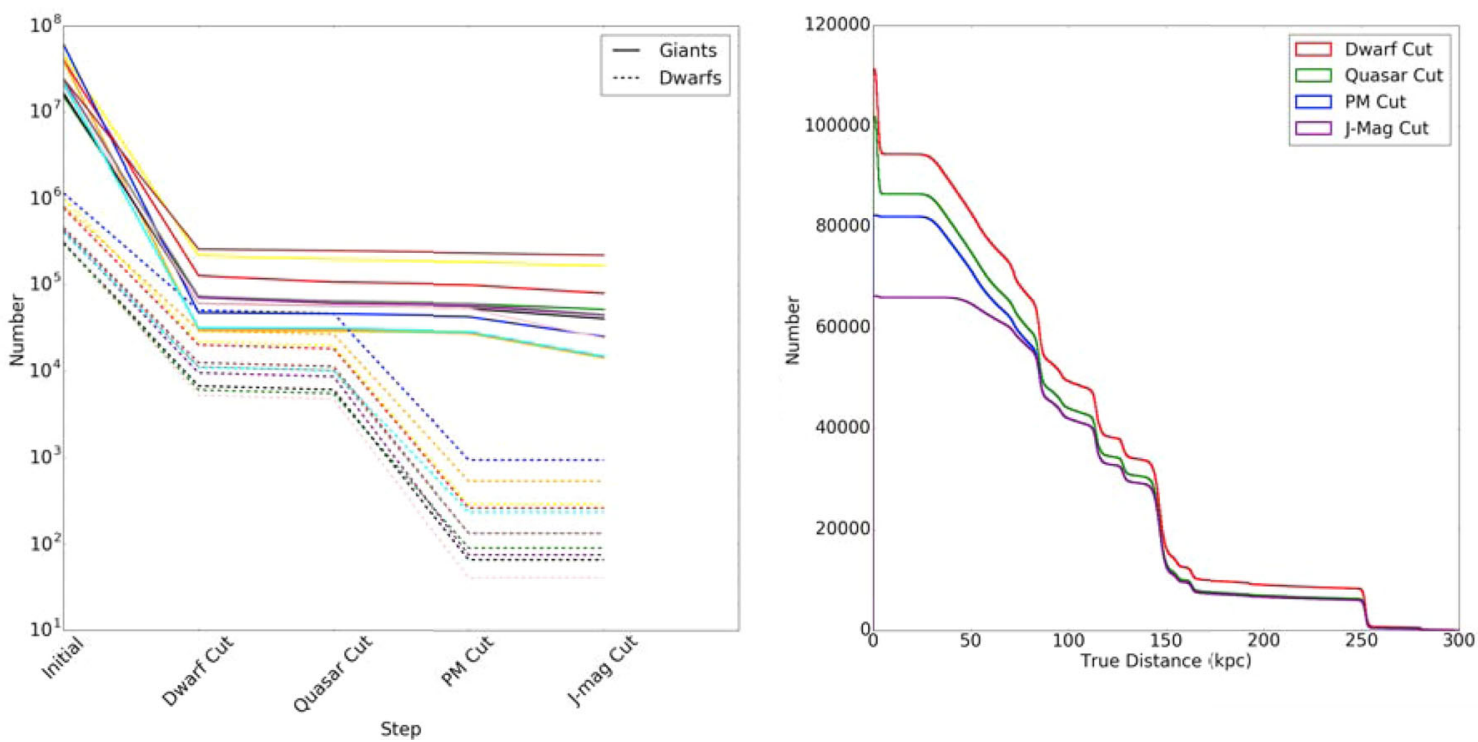

Figure 3. Left: the number of $M$ giants (represented by the solid lines) and dwarf stars (represented by the dashed lines) remaining in the error-convolved sample after each cut is made. Each colour represents a different mock halo. Right: the cumulative, from the outside in, average number of stars over all 11 error-convolved samples as a function of true distance remaining after each additional cut is made. In this panel, the different colours represent the successive selections on the sample, made in the order outlined in Section 3.1.2.

and out of selection colour space in most cases by a few per cent of the number selected for 'perfect' data. In 9 of the 11 haloes, the net effect of convolution is to scatter stars out of the selection box; in only two cases the net effect is to scatter stars into the selection. The per cent of the error-convolved and selected sample that is self-contaminating halo dwarf stars $(\log g>4)$ varies widely across the 11 mock haloes, from 4 per cent to just over 50 per cent, with about 15000 halo dwarf stars on average scattered into our colour space selections (ranging from just under 5000 to nearly 50000 ; see Fig. 3). These stars are generally at a heliocentric distance of around $2 \mathrm{kpc}$, and all are at a distance of less than $6 \mathrm{kpc}$, so they should be relatively easy to distinguish by their large PMs. Overall, we find that the colour-colour selections used by Bochanski et al. (2014a) are indeed successful at cutting down on the number of foreground dwarf stars, although they also throw out quite a few giant stars scattered in by photometric errors.

Next, we apply a PM selection as in Bochanski et al. (2014a), removing stars with PMs larger than $2.5 \sigma_{\mu}$ in either RA or Dec., with an average PM error of $\sigma_{\mu}=2 \mathrm{mas}^{-1} \mathrm{yr}$ as determined for the SDSS-USNO-B cross-match used in that work. This cut removes stars with high PMs, which are assumed to be in the foreground and therefore more likely to be dwarf than giant stars.

In contrast to Bochanski et al. (2014a), who found the PM cut to have little effect on their final sample, we find that this selection can have a large effect, removing anywhere from 9 up to 50 per cent of colour-selected, error-convolved stars that from Fig. 3 appear to be primarily dwarf stars within $100 \mathrm{kpc}$. We initially thought that this was an effect of the UKIDSS target area being out of the disc plane, while we make our PM cut on the entire sky. Even though our mock haloes do not include a stellar disc, the density of halo stars is higher in the plane of the disc-shaped part of the global gravitational potential, and we expected most of the removed stars to be in this plane that would not be covered by UKIDSS. Subsequent checks showed that this was part of the cause of this difference but not all of it; some of our mock haloes still have a significant amount of foreground removed by the PM cut even when only a high-latitude box is considered. We do not think this difference should be taken too seriously at this point for a few reasons. First, our mock haloes do not seek to directly reproduce the stellar density profile of the MW's halo, and these density profiles have been shown to vary substantially from galaxy to galaxy in reality anyway so some differences are to be expected. Secondly, the stellar population prescriptions used to generate the mock haloes are only approximate so some difference in absolute numbers of stars is to be expected especially along the giant branch where the models are not as well characterized. Thirdly, the halo still contributes only a small number of foreground stars compared with the disc foreground that is not included in our models. Given these caveats, we will defer further investigation of specific causes of the discrepancy, while emphasizing that especially given near-term improvements in PM accuracies from Gaia, our models do support the notion that PM cuts offer a powerful way to remove foreground stars.

Once these stars are removed, the per cent contamination by halo dwarf stars decreases dramatically to between 0.06 and 2 per cent, with an average among the haloes of 250 dwarf stars remaining and a range from 40 stars to just under 1000. On average, these stars are at a heliocentric distance of around $3 \mathrm{kpc}$, and all are at distances less than $6 \mathrm{kpc}$. The PM selection, when combined with the colour cuts, is therefore effective at removing the self-contamination of halo dwarf stars in the foreground even when the PMs are not measured very accurately. In the near future, cross-matching WISE, SDSS and 2MASS observations, and adding Pan-STARRS when available, could provide a longer baseline and become even more useful for removing foregrounds (including the halo as well as the disc).

The last step in the selection process is to select stars with simulated, error-convolved $J$ apparent magnitudes greater than 13.41 , the $J$ magnitude of the brightest star for which Bochanski et al. (2014a) obtained spectra. This magnitude limit is intended to remove any remaining foreground so we can focus on the truly distant stars, though there is not much of this in our case since we do not include a disc.

The right-hand panel of Fig. 3 shows that the final apparentmagnitude cut, represented by the purple line, is effective at 

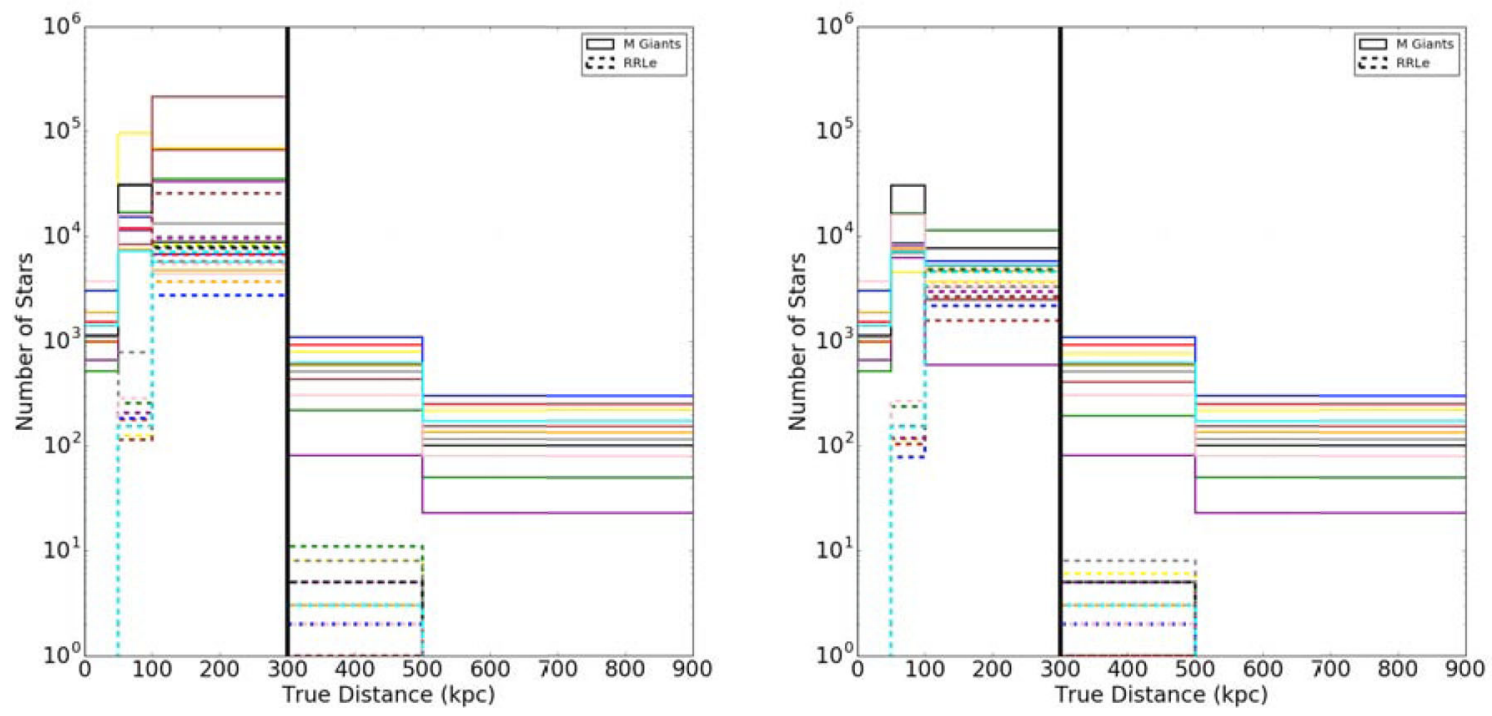

Figure 4. Number of selected $M$ giants (solid lines) and RRLe (dashed lines) for each of the mock haloes as a function of true distance for all substructures (left) and excluding still-bound satellites (right). Each colour represents a different mock halo. The dashed vertical line represents the virial radius.

removing nearby stars. After this selection is made, 64 per cent of the remaining stars are more distant than $100 \mathrm{kpc}$, and nearly 98 per cent are more distant than $50 \mathrm{kpc}$. After the full selection process, roughly 10000 to 300000 stars remain per mock halo, with a median value of around 50000 (Fig. 3, left-hand panel).

\subsection{A synthetic survey in RRLe}

In this section, we describe how we generate synthetic surveys of RRLe based on projections for LSST. VanderPlas \& Ivezič (2015) have demonstrated that RRLe can be perfectly identified over the entire LSST footprint down to magnitude 24.5 after a few years of operation, so the elaborate series of selections we used to separate the $\mathrm{M}$ giants from contaminating $\mathrm{M}$ dwarfs is not necessary for this tracer. Since LSST's footprint spans more than 3/4 of the sky, we use GALAXIA to generate a synthetic all-sky survey containing only RRLe at $100 \mathrm{kpc}$ and beyond, using the code's built-in option to sample RRLe only by analytically defining the location of the instability strip. The assumptions for the location and parameters of the instability strip model are given in section 2 of Sharma et al. (2011a).

Exquisitely accurate ( 2 per cent) distances can be obtained for RRLe by linking the optical light curves to infrared luminosities, where there is less scatter in the period-luminosity relation, which would in principle be possible by cross-matching with e.g. the HighLatitude Survey (HLS) planned for WFIRST, which will observe a contiguous area of about $2200 \mathrm{deg}^{2}$ in the infrared down to the $H$ band. The idea would then be to identify and fit light curves to RRLe in the HLS field using LSST, and then use WFIRST's infrared observations to obtain accurate distances. We tried simulated distance errors of 2 and 5 per cent and found few significant differences in the overall samples, so we have conservatively assumed 5 per cent distance errors. As with $\mathrm{M}$ giants above our primary interest is in the distant halo and so, using the error-convolved distances, we select only RRLe between $100 \mathrm{kpc}$ and the virial radius of the mock halo $(282 \mathrm{kpc})$. Thanks to the excellent distances, this selection does not eliminate very many stars from any of the synthetic RRL surveys.

\subsection{Distance distributions}

Fig. 4 takes a first look at the full size and extent of the M giant and RRL populations in the models by plotting the number of selected stars as a function of their true distances. $M$ giant stars in the mock surveys have a large distance range since we make no explicit distance cut when selecting them. There are over $1000 \mathrm{M}$ giants present beyond $100 \mathrm{kpc}$ for all 11 mock haloes and over 10000 for several of the haloes. There are also more than $1000 \mathrm{M}$ giants from tidally disrupted galaxies present beyond $100 \mathrm{kpc}$ for all but one of the mock haloes.

Because the distance errors are small for RRLe, most of the selected stars in this tracer fall within 100 and $300 \mathrm{kpc}$, with less than a thousand falling above or below this range for each mock halo. All but one of the mock haloes have between 1000 and 10000 RRLe between this distance range, with one halo having just over 10000 stars, and all having between 1000 and 10000 RRLe in tidally disrupted galaxies within this distance range.

When including stars in both still-bound satellites and unbound structures in these star counts, as in the left-hand panel of Fig. 4, there is over an order of magnitude in variation between the 11 GALAXIA haloes in the number of selected $\mathrm{M}$ giants or RRLe between 100 and $300 \mathrm{kpc}$. This variation decreases to around 0.5 dex when only unbound stars are plotted, as in the right-hand panel. This points to the star counts for some of the simulated haloes being dominated by dense, still-bound satellites within certain distances, whereas other haloes have fewer still-bound satellites in this distance range. The exception to this would be Halo 10, represented in purple, which appears to have roughly an order of magnitude fewer unbound $\mathrm{M}$ giants beyond $100 \mathrm{kpc}$. This is likely because most of the $\mathrm{M}$ giants (over 70 percent) in Halo 10 were accreted in a few massive objects about 4 Gyr ago, which have not yet had time to tidally disrupt. The time of accretion of our selected $\mathrm{M}$ giants over all 11 haloes peaks at 8 Gyr ago (see Fig. 8), which allows more structures to become unbound on average than in Halo 10.

Accreted building blocks that are still self-bound at the present day (i.e. satellite dwarf galaxies of the MW) are orders of magnitude smaller on the sky and more regular in shape than those that have been tidally disrupted, and are therefore easier to identify in stellar surveys. Fig. 4 also shows that the distance distribution of stars 

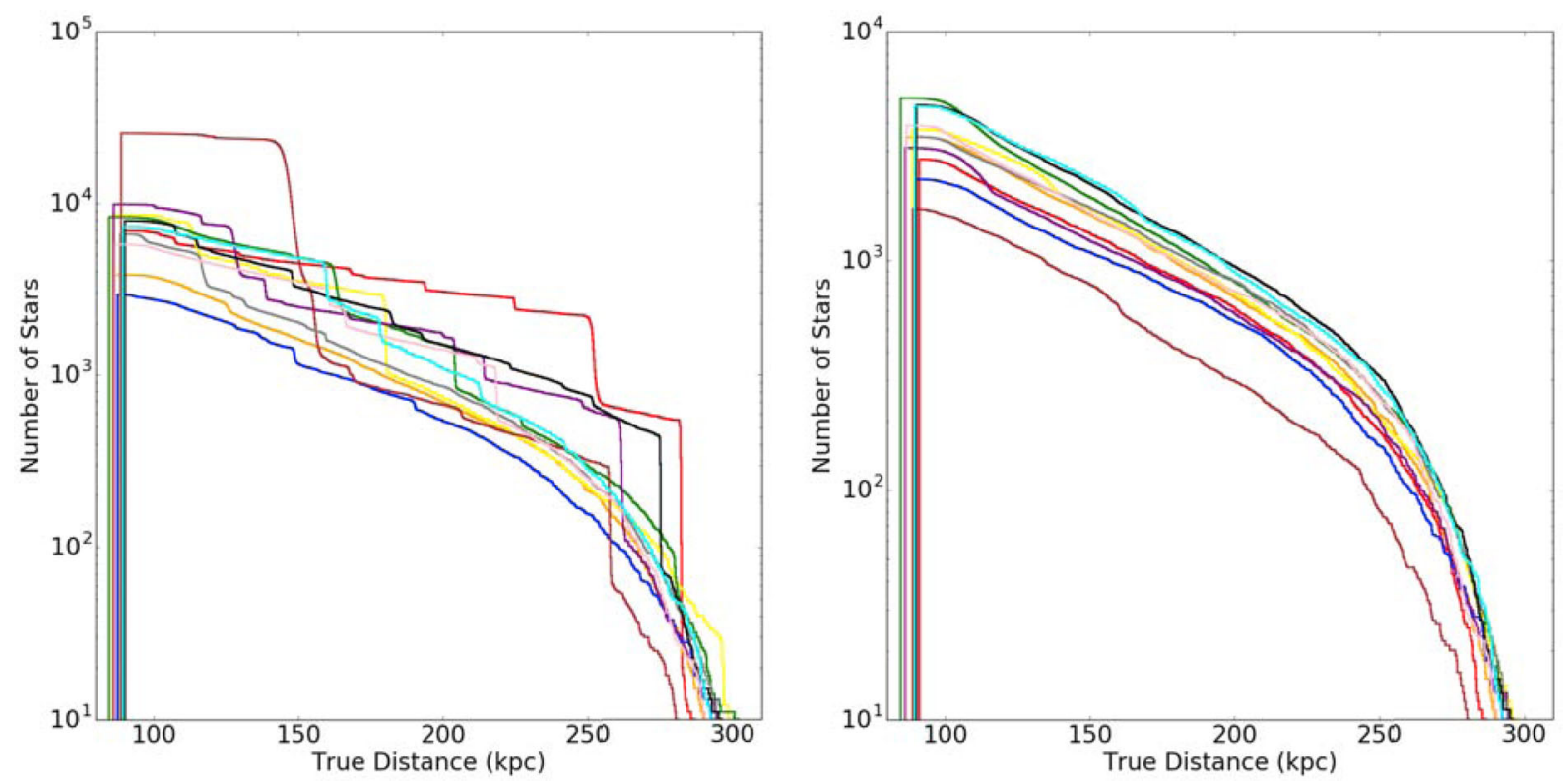

Figure 5. Cumulative, from the outside in, number of RRLe between 100 and $282 \mathrm{kpc}$ in error-convolved distances beyond each true distance as a function of true distance for all substructures (bottom left) and excluding still-bound satellites (bottom right).

in satellite galaxies also varies more from halo to halo than that for stars in tidal debris. For these reasons, we will find it useful to distinguish between stars in 'still-bound satellites' and stars in 'unbound structures', respectively, in the discussion that follows.

Comparing the two tracers, there appear to be more selected $\mathrm{M}$ giants than RRLe from still-bound satellites in the true distance range of $100-300 \mathrm{kpc}$, by up to an order of magnitude for several haloes. However, there are similar numbers of stars in unbound structures in both tracers in this distance range. This suggests that the $\mathrm{M}$ giant view of the stellar halo tends to be more dominated by stars in still-bound satellites. It is apparent that at least an order of magnitude more selected $\mathrm{M}$ giants fall below this distance range, because the variation in absolute magnitude of $M$ giants makes an apparent-magnitude limit imperfect at eliminating all nearby stars, especially compared to the small distance errors involved in RRL observation.

Thanks to the exquisite quality possible for RRL distance measurements, we can also consider the cumulative distance distribution of selected RRLe as a function of true heliocentric distances (Fig. 5), both for all substructures (left) and excluding still-bound satellites (right). Beyond $100 \mathrm{kpc}$ for 10 out of the 11 haloes, there are between 2000 and 10000 RRLe in total, of which between 1000 and 10000 are in unbound structures. Halo 12, represented in brown, is still a bit of an outlier with a greater number of bound stars and fewer unbound stars than is typical. As for M giants, the cumulative distance distribution becomes very consistent among the different haloes when excluding stars in still-bound satellites. In both the M giant and RRL tracers, we can draw the overall conclusion that if our models are representative of the MW, there are likely thousands of observable stars beyond $100 \mathrm{kpc}$, and that the distance distribution of tracers from unbound structures is fairly similar across all the mock haloes.

\subsection{Sky distributions}

As expected for a purely accreted population, the 11 mock haloes demonstrate a broad variation in the number and type of substruc- tures present at large distances, and this variation can differ for the two different tracers considered. This point is illustrated by comparing Figs 6 and 7, which show all-sky maps in Galactic coordinates of $\mathrm{M}$ giants and RRLe, respectively, for all 11 mock haloes. Each point in these two figures represents a selected error-convolved tracer colour-coded by the building block it belongs to; the same colour is used for the same building block in each halo across both figures.

In most of the mock haloes, it is apparent that stars are not evenly distributed across the sky, but there is a good deal of variation. Halo 14 , for example, is dominated by one diffuse unbound structure in $\mathrm{M}$ giants at central latitudes (represented in yellow), and then several somewhat less diffuse, still-bound satellites at higher latitudes. In RRLe, the diffuse yellow structure is much less apparent while some of the smaller structures (like those coloured blue and purple) stand out. Alternatively, Halo 2 has a broad, diffuse distribution of $\mathrm{M}$ giants at central latitudes comprised of many unbound structures, which is echoed in the RRL distribution albeit with fewer tracers overall. The $\mathrm{M}$ giant map of Halo 17 shows several long distinctive stellar streams (in purple and dark yellow for example), while these are almost completely absent from the RRL map of the same halo. Finally, Halo 20 provides two particularly good examples of $\mathrm{M}$ giants that were accreted as part of the same building block but are now located in unbound groupings at opposite ends of the halo. These two large structures (in purple and pink in the $\mathrm{M}$ giant map of Halo 20 shown in Fig. 6) form overdensities despite being unbound. Only one of the two structures is easily visible in the RRL map of the same halo.

The RRL tracer also shows significant variation between haloes. Halo 12, the biggest outlier in Fig. 5, has several still-bound satellites that contain most of the mock halo's stars, while Halo 15 has mostly unbound structures. Halo 9 is relatively empty at high latitudes, whereas Halo 10 has two large dense structures at high latitude, and Halo 8 has a heavy distribution of stars all over. As for M giants, stars accreted together can span hundreds to thousands of square degrees and end up on opposite ends of the halo.

The RRL haloes are more sparsely populated than the M giant maps, both due to there being a larger predicted number of $\mathrm{M}$ giants 

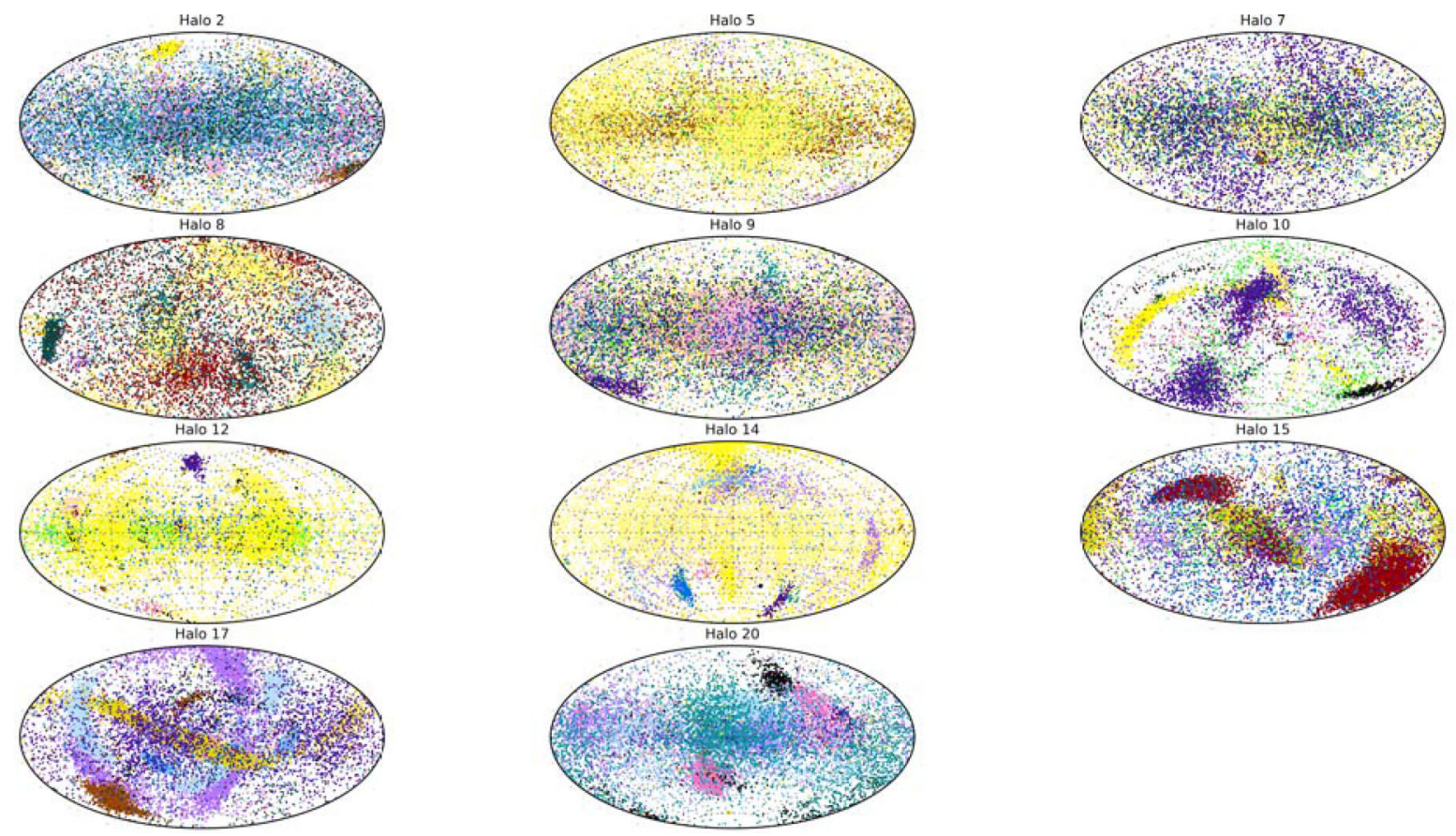

Figure 6. All-sky map of M giants selected as in Section 3.1.2 for each of the 11 mock stellar haloes. Different colours represent different building blocks.
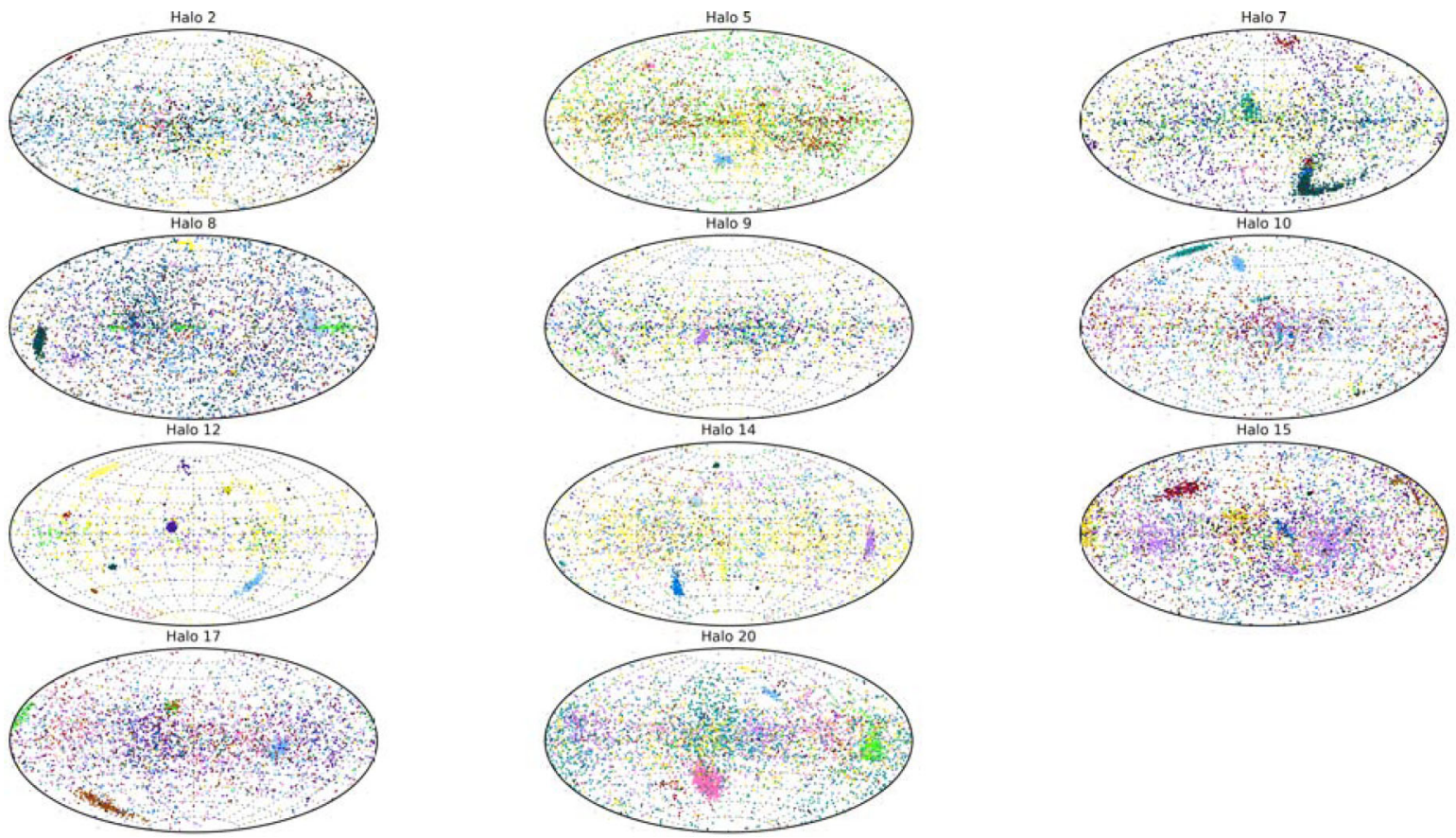

Figure 7. All-sky map of RRLe between error-convolved distances between 100 and $282 \mathrm{kpc}$ for each of the 11 stellar haloes. Different colours represent different building blocks.

and to the apparent-magnitude cuts made for the $\mathrm{M}$ giants that do not eliminate as many stars outside the more specific distance range set for the RRLe. The greater number of $M$ giants represented in Fig. 6 than in Fig. 7 leads to several structures being more clearly defined in the M giant tracer than in the RRL tracer, such as the large red structures in Halo 15, which barely appear in the RRL tracer but which are extremely prominent in the $\mathrm{M}$ giant tracer. The looser distance cuts in the $\mathrm{M}$ giant tracer also lead to more stream-like structures being picked out in Fig. 6 than in Fig. 7 such as those in Halo 17 and those represented in yellow in Halo 10. Despite the greater number of $M$ giants, there are still a few structures only picked out in the RRL tracer as mapped in Fig. 7 such as the light blue and turquoise structures in the upper latitudes of Halo 10 and the dark green structure in the lower latitudes of Halo 7.

To summarize, our models suggest that there are around 1000 to 10000 unbound $\mathrm{M}$ giants beyond $100 \mathrm{kpc}$, in other words less than one per square degree on average, and a comparable or slightly smaller number of RRLe in this distance range. These stars are 

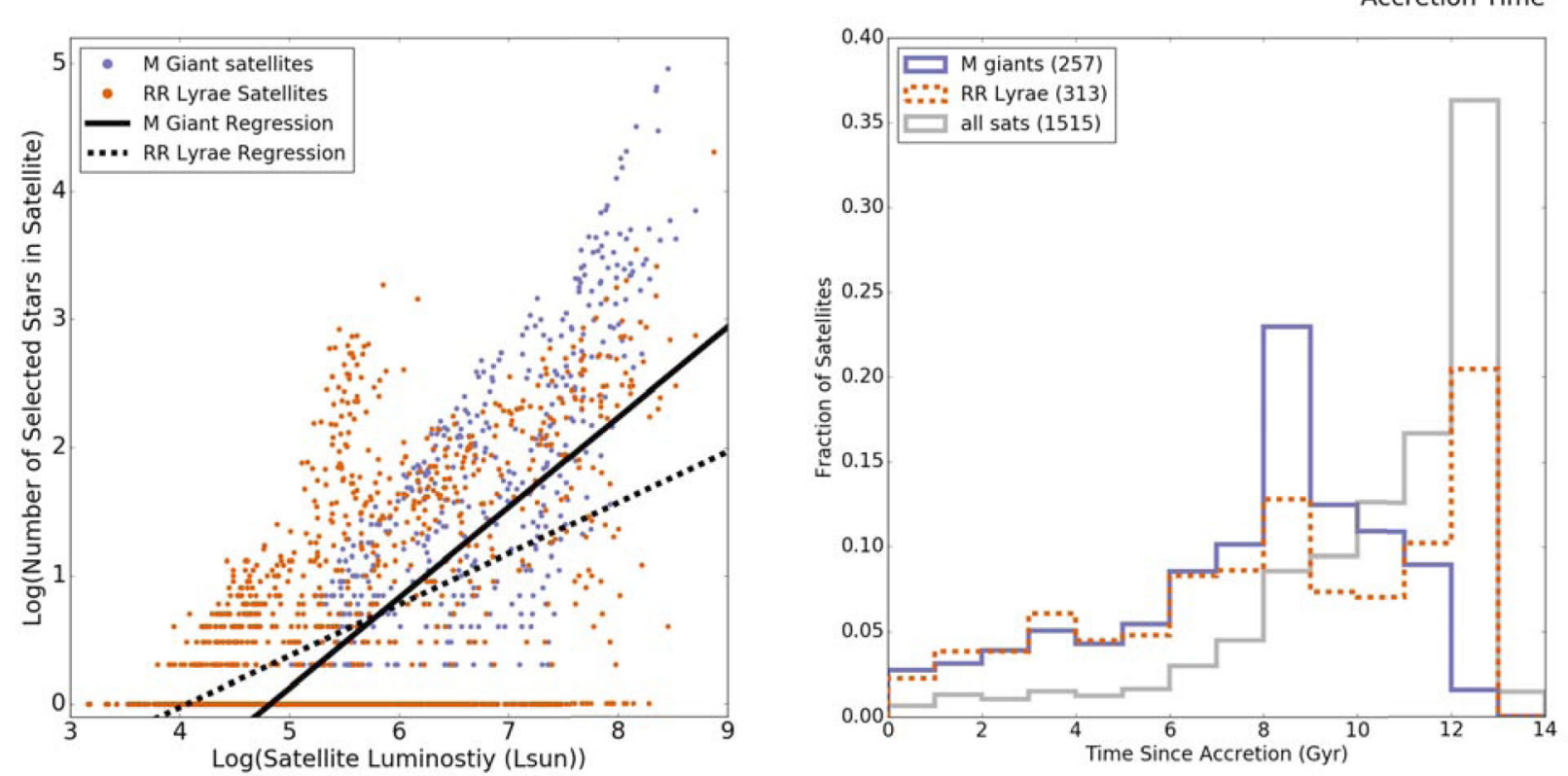

Luminosity
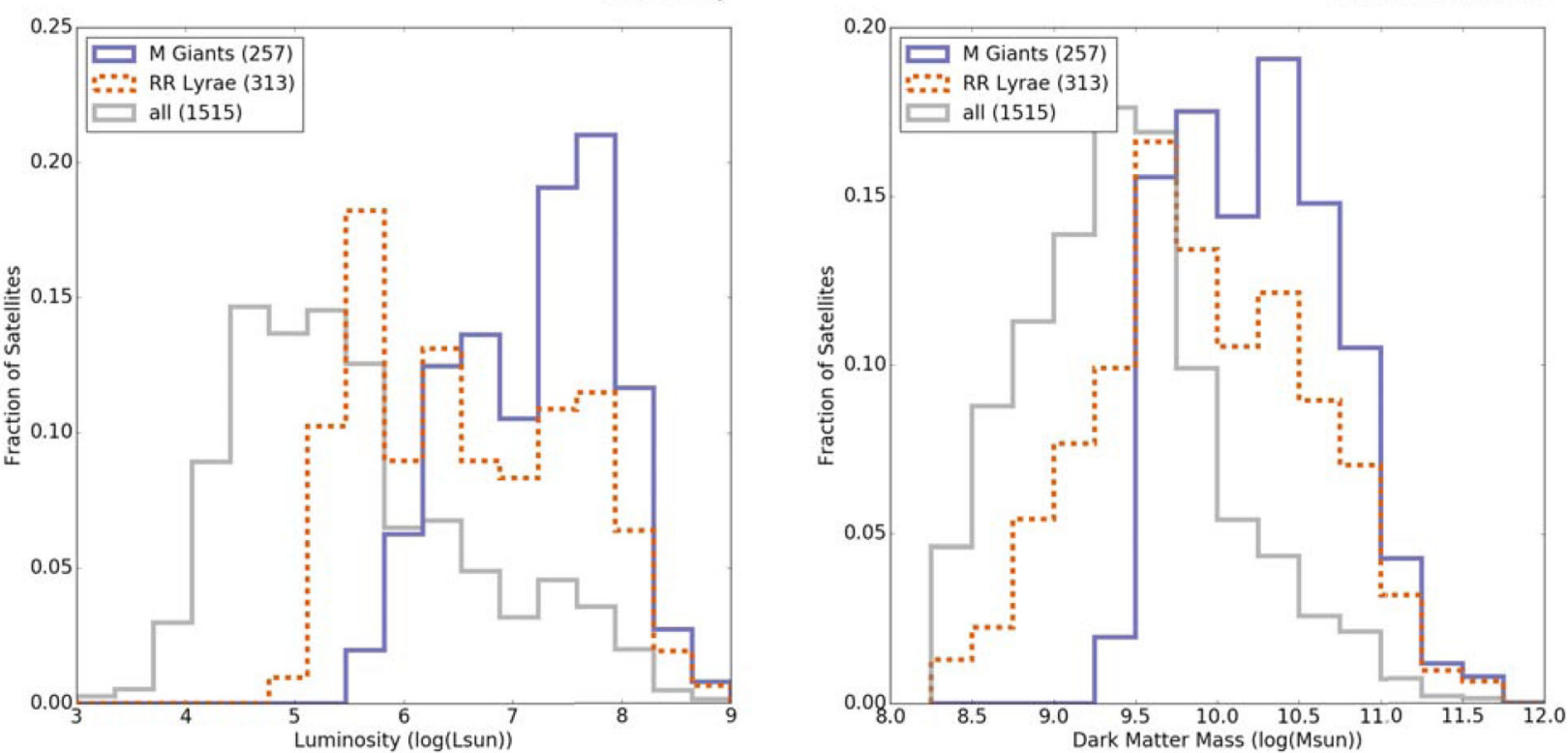

Figure 8. Top left: logarithmic distribution of total building block luminosity versus number of selected M giants (blue) and RRLe (orange) in that building block. Other panels: distribution of various properties of building blocks containing 20 or more selected M giants (solid blue lines) or RRLe (dashed orange lines) compared to the overall distribution of building blocks (grey). Top right: time since accretion; bottom left: total luminosity; bottom right: total dark matter mass.

distributed non-uniformly across the sky, and this distribution varies significantly between haloes: in some haloes even the unbound structures are fairly dense and compact, while in others the accreted structure is spread over much broader areas of sky. In addition, associated debris can cover hundreds to thousands of square degrees in structures, sometimes on opposite sides of the sky.

\subsection{Distribution of tracers among the accreted building blocks}

Now we examine the properties of the building blocks containing the different tracers selected by our observational cuts. In the top- left panel of Fig. 8, we compare the number of M giants (blue points) or RRLe (orange points) in a building block to the total luminosity of that building block. We find that for building blocks containing more than about $10 \mathrm{M}$ giants, building block luminosity is strongly correlated with the number of $\mathrm{M}$ giants associated with that building block. There is also a slight positive correlation between the number of selected RRLe in a building block and total luminosity, but with slightly more scatter at a given luminosity. Since more RRLe form in metal-poor populations, even the lowest luminosity building blocks contain a few, and so the entire distribution is shifted towards lower luminosities for RRLe. 

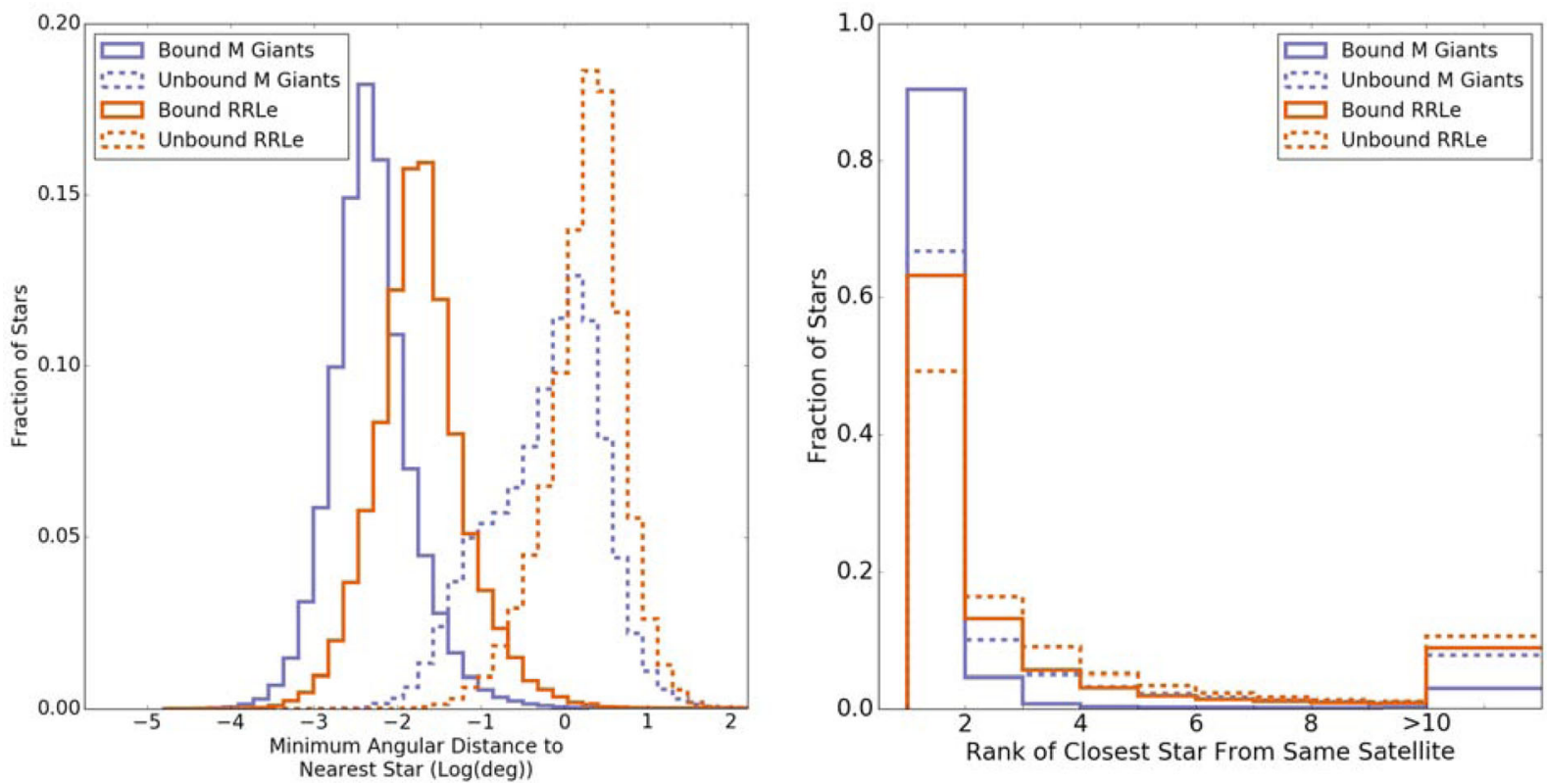

Figure 9. Statistics of nearby pairs of stars from two tracer populations: M giants (blue) and RRLe (orange). Building blocks that are still bound at the present day are shown with solid lines, and building blocks that are now tidally disrupted are shown with dashed lines. Left: distribution of the minimum angular distance (in $\log ($ degrees $)$ ) to the nearest tracer from the same population that was accreted as a member of the same building block. Right: distribution of the rank in distance of the closest tracer from the same population in the same building block: 1 if the closest tracer is in the same building block, 2 if the second closest tracer is the same building block but the first closest is not, and so forth. These distributions are for all selected tracers in the two populations, in all 11 mock haloes.

For building blocks containing only a few M giants, there is a very large range of luminosities, as much as three orders of magnitude. These trends come from the assumptions in the models that infalling dwarf galaxies follow a stellar mass-metallicity relation. Since giant stars only become red enough to reach $\mathrm{M}$ spectral type if their metallicity is above $[\mathrm{Fe} / \mathrm{H}] \sim-1$, then the number of $\mathrm{M}$ giants can be expected to loosely track the overall luminosity of a building block, but cannot be used to make any precise assumptions about the total luminosity of the dwarf galaxy before infall. Metallicity measurements for the individual $\mathrm{M}$ giants in a structure, however, could help break this degeneracy as well as potentially helping to disentangle different accreted structures from one another, as we will discuss briefly in Section 7.

As we pointed out in Section 3.1.2, the colour selections we made for the $\mathrm{M}$ giants tend to favour metal- and alpha-rich stars, while RRLe are more abundant in metal-poor populations. In the remaining three panels of Fig. 8, we examine how this affects the types of building blocks that are prominent in the two tracers. We compare the properties of building blocks containing a minimum of 20 selected tracers (i.e. structures that could potentially be identified and characterized) to the overall distribution of halo building blocks for all 11 haloes combined. We observe that building blocks containing 20 or more $\mathrm{M}$ giants tend to be more luminous and have a greater dark matter mass, compared to the general population, while building blocks containing 20 or more RRLe track slightly less luminous and less massive objects. Building blocks picked out in $\mathrm{M}$ giants also tend to have been accreted more recently than the general population, peaking between 8 and $9 \mathrm{Gyr}$ ago as opposed to around 12 Gyr ago (top-right panel of Fig. 8); those picked out in RRLe track more closely to the overall distribution of accretion times but still are still skewed towards slightly more recent accretions. The preference for later-accreted, more massive objects is likely an effect of requiring building blocks to have at least 20 trac- ers, an effect that is stronger for the $\mathrm{M}$ giants since our selection box prefers more metal-rich stars. This effect dominates over the tendency of the more massive building blocks to migrate towards the centre under the influence of dynamical friction. Only for the most massive building blocks is the migration time-scale fast enough to matter; otherwise, it depends on the accretion time. The preference for more recently accreted building blocks also reflects our choice to study the outer halo; in this cosmological model, more distant building blocks tend to be more recently accreted. (See also Sharma et al. 2011a, for a related discussion.)

\section{FINDING BUILDING BLOCKS OF THE ACCRETED HALO}

We now consider the prospect of finding individual building blocks by searching the two tracer distributions. One possible approach to searching for structures is simply to search for close pairs of tracers from the same stellar population. Sesar et al. (2014) and Baker \& Willman (2015) use this approach to search for new dwarf galaxies orbiting the MW in samples of RRLe, so we investigate whether this is also an effective technique for $\mathrm{M}$ giants. We would like to determine whether tidally disrupted structures could also be identified with this method despite their much larger angular size compared to still-bound satellites.

The left-hand panel of Fig. 9 shows the distribution of angular distances to the nearest star of the same building block for the selected M giants (blue) and RRLe (orange) for all 11 haloes combined. In the right-hand panel of the figure, we show the distribution of the rank of the nearest star that was accreted in the same building block. This rank is 1 if the closest star from the same tracer population is in the same building block, 2 if the second closest star is the closest star that is in the same building block, and so on. In both panels, the distribution for M giants (RRLe) in still-bound satellites is shown 

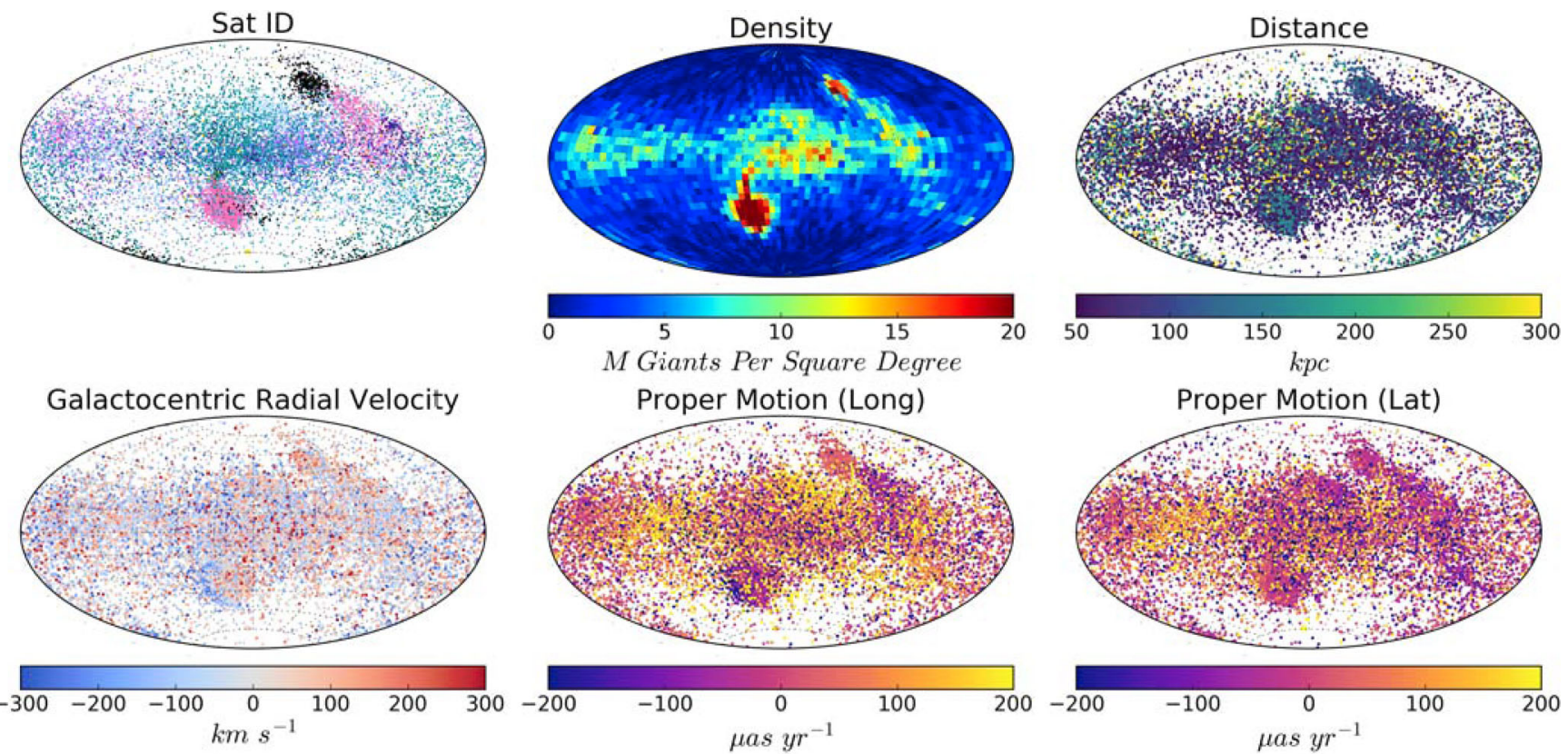

Figure 10. Six all-sky views of selected M giants for Halo 20. Each map is colour-coded by different properties: the satellite the star was accreted with (top left), the density, per square degree, of M giants from unbound structures (top centre), the heliocentric distance with 20 per cent errors drawn from a Gaussian (top right), the galactocentric RV of the star (bottom left) and the galactocentric PM of the star (longitudinal: bottom centre, latitudinal: bottom right).

as a solid line, and for unbound structures as a dashed line. In both scenarios, still-bound and unbound, and for both tracers, the closest star to each star in the same tracer population tends to be from the same building block. For $\mathrm{M}$ giants, this chance is around 70 per cent for unbound structures and around 90 per cent for still-bound satellites. The nearest RRL is from the same building block somewhat less frequently, roughly 60 per cent of the time for stars from still-bound satellites and roughly 50 per cent of the time for stars from unbound structures.

It is somewhat more likely that the nearest star from the same building block is the second through ninth closest star to a particular $M$ giant in the unbound case than in the still-bound case. Less than 10 per cent of the time will the nearest $M$ giant from the same still-bound satellite be greater than the second closest star, and the same is true for the fifth closest star for a member of an unbound structure. The fraction of stars from the same satellite that are more than 10 stars away from the nearest star they were accreted with is also shown. This appears to be more common for stars from unbound structures but still occurs less than 10 per cent of the time for $\mathrm{M}$ giants. It is more common (over 10 per cent of RRLe) for the nearest RRLe from the same building block to be more than 10 stars removed, for both the still-bound and unbound cases, than for the nearest $\mathrm{M}$ giant from the same satellite to be over 10 stars away.

That stars closest to each other in our data tend to have been accreted from the same building block, even if they have since been tidally disrupted, is a promising development for being able to identify different building blocks in the stellar halo. We further find that the characteristic distance between tracers in a given building block can be used to differentiate between still-bound satellites and unbound structures. The left-hand panel of Fig. 9 helps to provide a diagnosis, when two stars from the same tracer population are discovered near to each other, as to whether these stars are part of a still-bound or unbound structure. The distribution of distances between $\mathrm{M}$ giants for still-bound satellites clearly peaks around a few arcseconds, while the distribution for the unbound structures clearly peaks around a few degrees, with little overlap. RRLe tend to be somewhat farther from their nearest neighbour in the same building block, with the RRL distribution peaking at around an order of magnitude larger angular distance than the M giant distribution. For the unbound structures, although distributions for both tracers peak at around a few degrees, nearly a 10th of $\mathrm{M}$ giants are located less than a 10th of a degree from their nearest neighbour from the same building block, versus only a couple per cent of RRLe.

The angular distance between two observed $\mathrm{M}$ giants could potentially be used to determine whether they belong to a still-bound or unbound building block, although doing so would require excellent foreground removal (remember that we do not include the disc foreground in our models). The distribution of distances also points to the need of contiguous observational fields covering at least a few square degrees to have a good chance of locating two stars in the same tracer population from the same unbound structure. Baker \& Willman (2015) have performed a more extensive test of the ability to detect still-bound satellites using pairs of RRLe with physical linking lengths in two dimensions in a series of bins in distance modulus; our finding lends support to their approach. Furthermore, although Baker \& Willman focused on finding dwarf galaxies and used an appropriately small linking length, the same technique with a larger link length could potentially also identify unbound structures. Additionally, for a survey with limited field size, our models suggest that it is not only easier to locate multiple $\mathrm{M}$ giants together, but also that the $\mathrm{M}$ giants are more likely to be from the same building block, whether or not they are still bound to it.

\section{DISTINGUISHING DIFFERENT ACCRETED STRUCTURES}

In this section, we investigate what information is most useful to untangle different accreted structures that overlap each other on the sky. In Fig. 10, we view an all-sky map of the selected M giants of an example halo, Halo 20, colour-coded by six different properties: 
satellite the star was accreted with; stellar density, binned by square degree and only including unbound structures; heliocentric distance with 20 per cent errors drawn from a Gaussian; galactocentric RV; galactocentric longitudinal PM; and galactocentric latitudinal PM. Stellar overdensities (top centre) clearly occur at locations where even unbound structures are present, and in most of the stellar halo there appears to be at least one selected $\mathrm{M}$ giant present per square degree, especially at lower galactic latitude. Close examination of the longitudinal and latitudinal PM all-sky maps (bottom centre and right) shows that both components of a star's PM can be used to differentiate between overlapping structures, as stars that are in close proximity to each other in galactic latitude and longitude, but not belonging to the same accreted satellite, tend to stand out from each other by having different PMs. In addition, stars from the same accreted satellite tend to have similar PMs. For example, the satellite that is dark pink in the top-left plot has a relatively

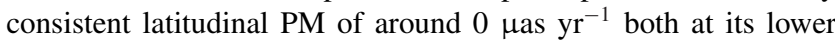
latitude and upper latitude positions. The many non-pink stars (i.e. stars from other accreted satellites) that are seen overlapping the pink satellite in the top-left panel also tend to be discernable from the pink satellite when looking at their latitudinal and longitudinal PMs in the bottom-right and bottom-centre plots. In the longitudinal PM plot (bottom centre), stars belonging to the satellites coloured in turquoise and light blue in the upper-left plot can be distinguished where they overlap the dark pink satellite at lower latitudes on the sky, because of the difference in average latitudinal PM.

Not every star's association can be specified using PMs alone: different satellites can still have similar PMs and there is some variation in PMs among stars belonging to the same satellite. However, for satellites that are distinguishable in the figure, the differences between the average PMs of different accreted structures seem to be of the order of $50-100 \mu \mathrm{as} \mathrm{yr}^{-1}$, whereas the spread within structures is often less than this. This indicates that PM measurements

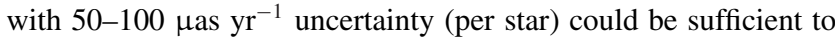
start to distinguish different tidal streams at these distances. This is significantly lower than current PM uncertainties, but Gaia and later LSST and WFIRST are expected to achieve this precision. The orbit distribution of the accreted satellites is fairly radial, so this is a fairly conservative estimate of the variation in PMs.

Differences between accreted structures are significantly less apparent in RVs (bottom left): the overlapping structures seem to have more intrinsic RV variation than PM. This is likely because many of the unbound structures at these radii are shells: that is, they are tidal debris in the process of piling up near apocentre. Material truly at apocentre will have zero RV, but this debris is bracketed on one side by outgoing material headed to apocentre and on the other by infalling debris that has already turned around, producing a distinguishable gradient in RV over a single structure. For example, the dark pink satellite that was somewhat uniformly coloured in the both PM plots has nearly the full range of RVs. This makes it very difficult to pick out the overlapping satellites, although the occasional overlapping star from a different satellite still does pop out, such as the two particularly red (high positive RV) stars in this region, which are members not of the pink satellite but of the one colour-coded as turquoise in the upper-left panel.

Although RVs are perhaps less useful for disentangling different structures at these distances in the halo, they are very useful for constraining the MW-mass profile, since the gradient in RV apparent for a few structures in Fig. 10 depends directly on the local radial acceleration (Merrifield \& Kuijken 1998; Sanderson \& Helmi 2013). Errors in the heliocentric distances of the selected M giants, as shown in the top-right panel of Fig. 10, make distance less useful for differentiating structures, even many of the unbound structures in Halo 20 are shells, where most of the material is at apocentre. Shells tend to be only a few kpc thick, far less than the distance errors in our model, which makes them difficult to distinguish. Instead, $\mathrm{M}$ giants that are members of the same accreted satellite, whether or not they are still bound, seem more likely to be more closely located in terms of latitude and longitude than in terms of radial distance. We conclude that if the MW accretion history resembles that of Halo 20, we can find unbound structures on the sky as overdensities, potentially disentangle overlaps using PMs and then use their RVs to constrain the MW-mass profile. For this analysis, we have so far considered a structure 'identifiable' if it has more than $20 \mathrm{M}$ giants, and we expect that this should be enough for a measurement of the enclosed mass inside a shell, although we will defer testing that assumption to future work.

We also consider which information is most useful to separate different structures from each other when viewed in RRLe. The most useful components may indeed be different, since for example we expect RRLe to have much better distance estimates than M giants. Six all-sky maps of the selected RRLe of Halo 20, analogous to Fig. 10, are presented in Fig. 11. They are colour-coded as following: satellite the star was accreted with (top left), stellar density binned by square degree and only including unbound structures (top centre), error-convolved heliocentric distance (top right), galactocentric RV (bottom left) and galactocentric PM (longitudinal: bottom centre, latitudinal: bottom right). Once again, we can clearly see overdensities where there are substructures, even if they are unbound. In particular, the satellite coloured in pink near the centre of the halo and to the right, as well as the bright green satellite concentrated towards the right of the map, and the light blue satellite concentrated at higher latitude appear to be picked out nicely as overdensities. As expected, compared with the density plot in Fig. 10, there are lower densities of RRLe present especially at lower latitudes.

In Fig. 11, both longitudinal and latitudinal components of PM seem to be more consistently closer to $0 \mu$ as $\mathrm{yr}^{-1}$ than for the $\mathrm{M}$ giants in Fig. 10. On the other hand, in particular the longitudinal component of PM of RRLe seems to be more distinctive among different accreted satellites than in the $M$ giant tracer, which is apparent when observing the difference in longitudinal PM (bottom centre) between the RRLe that are members of the satellite colourcoded in pink in the top-left plot and the other overlapping satellites.

The much smaller errors in distance for RRLe lead to different structures being more uniformly coloured in the distance plot (top right), suggesting that in this tracer determining distances may prove more useful in differentiating between structures than for $\mathrm{M}$ giants. On the other hand, there is still variation in the RVs of RRLe from the same satellite, again because these structures are mostly shells. Overall, Fig. 11 shows that, as with M giants, overdensities can help pick out structures that can then be differentiated by PM components, perhaps even more effectively than in the $\mathrm{M}$ giant tracer and, due to smaller distance errors, by distance as well.

\section{EXPECTATIONS FOR A UKIDSS-LIKE SURVEY}

We now examine the $\mathrm{M}$ giant view of the distant stellar halo in the context of the current UKIDSS, which subtends about $2200 \mathrm{deg}^{2}$ of contiguous area on the sky. In our mock haloes, the Sun could be located anywhere on the solar circle (presumed at $8 \mathrm{kpc}$ ), so we randomly placed 500 UKIDSS-sized surveys at mid-latitude locations (between either 20 and 70 , or -20 and -70 degrees latitude) 

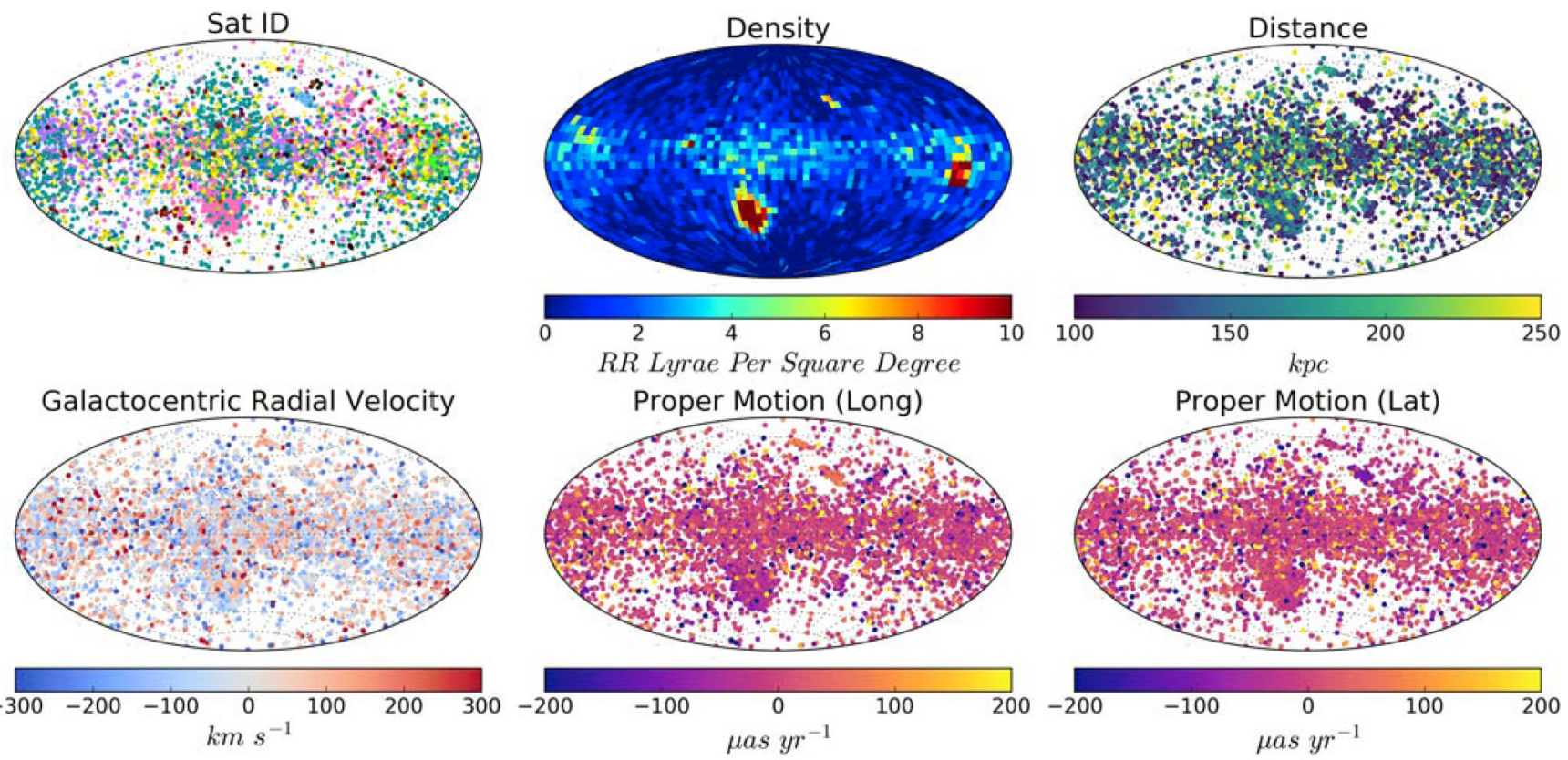

Figure 11. Six all-sky views of RRLe between error-convolved distances of 100 and $282 \mathrm{kpc}$ for Halo 20. Each map is colour-coded by different properties: the satellite the star was accreted with (top left), the density, per square degree, of RRLe from unbound structures (top centre), the error-convolved heliocentric distance (top right), the galactocentric RV of the star (bottom left) and the galactocentric PM of the star (longitudinal: bottom centre, latitudinal: bottom right).
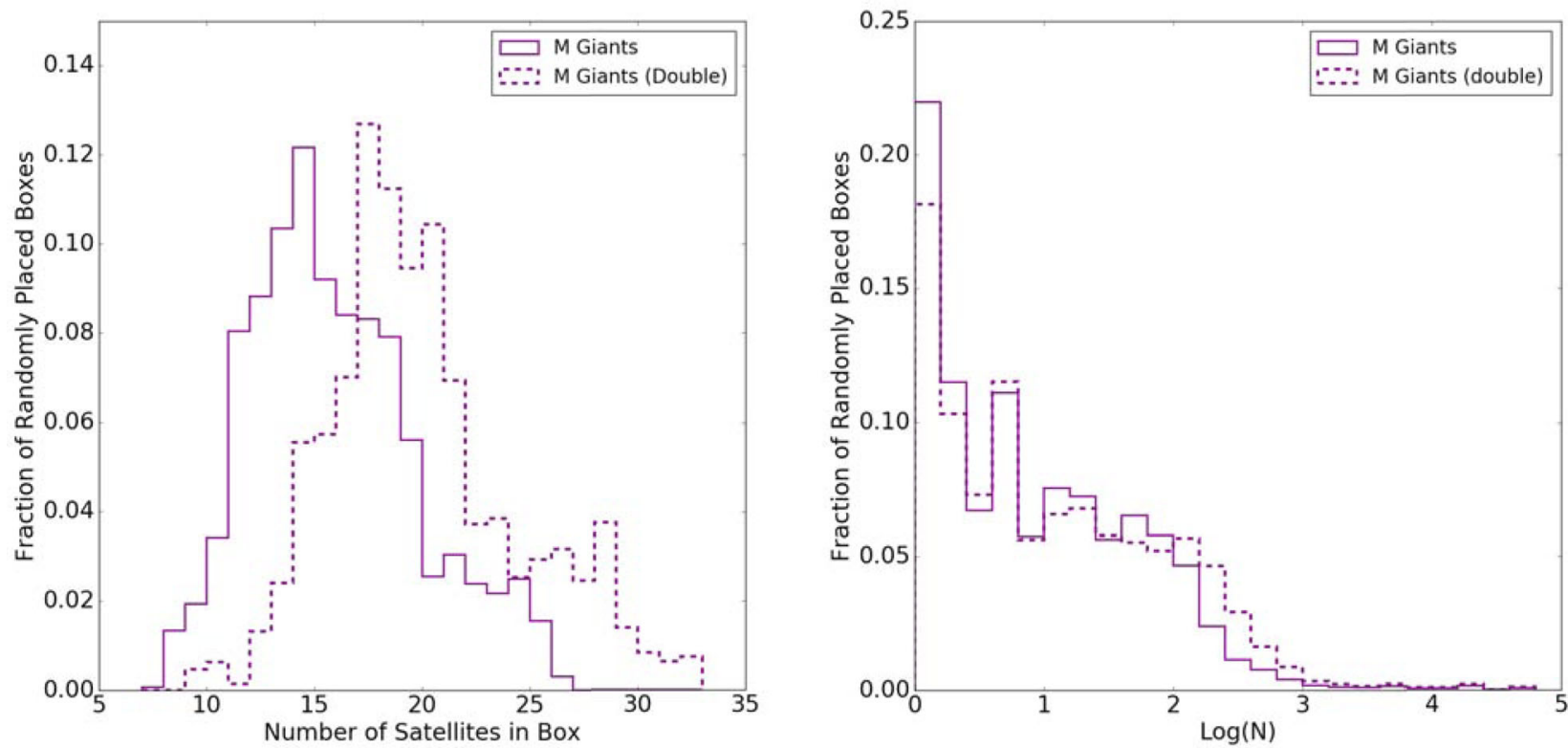

Figure 12. Left: distribution of the number of satellites containing $M$ giants in a randomly placed $2200 \mathrm{deg}^{2}$ survey (solid), and a survey twice the size (dashed). Right: distribution of the number of M giants from the same satellite that fall in a $2200 \mathrm{deg}^{2}$ survey (solid), and survey twice the size (dashed).

in each of the 11 mock haloes, for a total of 5500 randomly oriented survey fields. Strictly speaking, this is not geometrically equivalent to moving the Sun's location, but since we are focusing on distances beyond $50 \mathrm{kpc}$, the differences are slight. When done this way, we can also use the same sample to explore the effect of the location of the survey footprint. The solid lines in Fig. 12 show the distribution of the number of satellites containing selected $\mathrm{M}$ giants located in a survey this size (left), and how many $M$ giants per satellite we would find in the survey (right). Based on our 11 mock haloes, we find that a roughly $2200 \mathrm{deg}^{2}$ survey at mid-latitudes will always consist of multiple satellites, with a median of about 14 different building blocks represented. About 80 per cent of the time, at least one satellite will have more than one $\mathrm{M}$ giant star in the survey area; about half the time at least one will have more than 10 stars and rarely (about 10 per cent of the time) the survey box includes at least one satellite with more than $100 \mathrm{M}$ giants belonging to it. We therefore expect that a typical survey of this size will be dominated by one or two structures, but will contain a few stars from a good sample of the halo building blocks.

It is interesting to ask whether increasing the survey size has a significant effect. The dashed lines in Fig. 12 show the number of satellites and number of stars per satellite change if we 


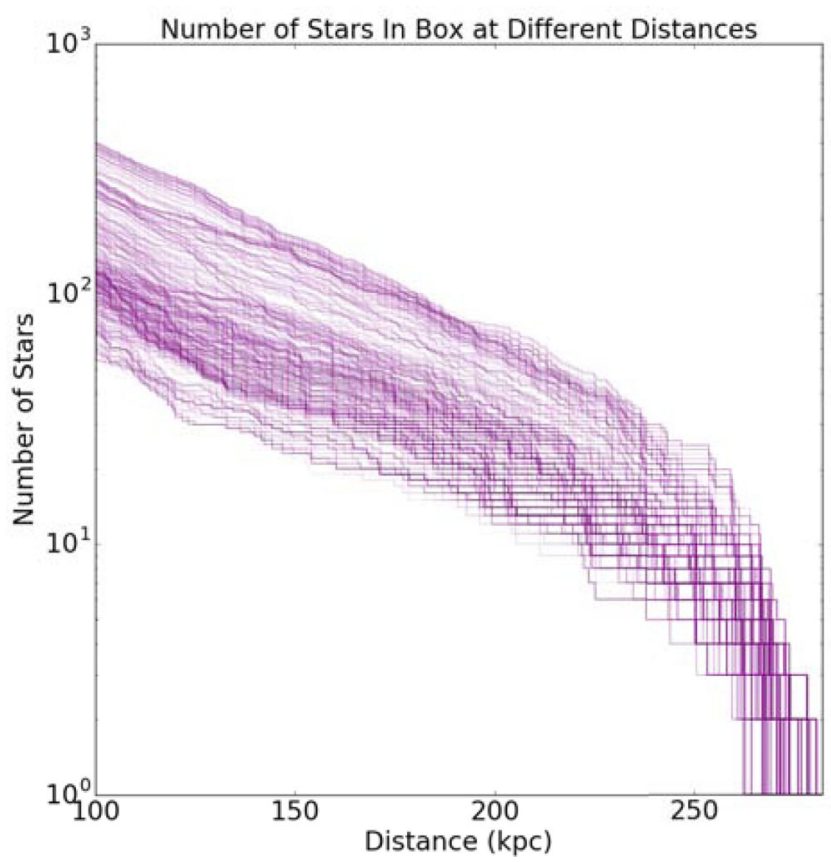

Figure 13. Number of $M$ giants (cumulative from the outside in) in 500 randomly placed $2200 \mathrm{deg}^{2}$ fields as a function of true distance, for Halo 5 .

double the contiguous survey area to $4400 \mathrm{deg}^{2}$. When expanding the survey area to twice the size, the most likely number of satellites contributing increases somewhat, from 14 to 18 , but the number of stars per satellite is not greatly affected except at the high end of the distribution (above $\sim 100$ stars). Doubling the sky coverage thus helps map out more completely the larger unbound, but still spatially associated structures, such as the dark red structure in Halo 15 in Fig. 6, as well as stream-like structures, such as those in Halo 17 in Fig. 6, and widely spread diffuse structures, such as the yellow structures in Halo 5 and Halo 14. Interestingly, the number of different satellites recovered in the doubled survey area is increased by less than a factor of 2 . This suggests that, in general, satellites in our simulations are not evenly spread across the sky, which is supported by the all-sky maps of Fig. 6 .

It is also interesting to ask how representative a given survey field of this size is of the global halo population. In Fig. 13, we show the effect of the location of the survey footprint on number of selected $\mathrm{M}$ giants of heliocentric distance for Halo 5, represented in orange in Fig. 4. Halo 5 was chosen because it does not have many bound structures, which we assume will be identified with confidence. We find that changing the location of the survey footprint produces less than an order of magnitude difference in the number of stars detected within a certain distance. The location-dependent spread is also comparable to (or perhaps even less than) the variance in the number of $\mathrm{M}$ giants between different haloes in the set. We conclude that a survey of a few thousand square degrees at mid-galactic latitudes should provide a fairly representative sample of the distant stellar halo. We do note that the primary variation of our field placement is with longitude (although we do consider fields both above and below the Galactic disc), and the Galactic mass model used here is axisymmetric, so any effects of halo triaxiality or filamentary accretion are not present in our mock haloes and could affect this result to some degree. However, the degree to which the MW halo is triaxial and the directions of any local filamentary flows are both only poorly constrained; in the absence of better information, we consider the axisymmetric assumption to be a reasonable one.

Presuming that our mock haloes resemble the MW's, the results of this section demonstrate that a survey field of a few thousand square degrees is both sufficient to get a general sense of the contents of the distant halo and is likely to contain well-sampled debris from about one to three accreted satellites that could be associated using additional dimensions of information (beyond sky positions). This bodes well for current searches for distant $\mathrm{M}$ giants based on UKIDSS like that described in Bochanski et al. (2014a); it means that the discovery of stars like the one featured in Bochanski et al. (2014b), at distances beyond $200 \mathrm{kpc}$, is expected, and that this star and others like it will likely turn out to be in the most well-sampled tidal structures. Finding even one well-populated accreted structure at large galactocentric distance would be a powerful probe of the Galactic potential. As the search continues, proximity of these very distant stars on the sky might provide one path to associating them (as we discussed in Section 4). In the future, deeper surveys of a similar size (like the WFIRST HLS) will push further, beyond the edges of the MW.

\section{RATIOS OF STELLAR POPULATIONS}

As noted by Bell et al. (2010), the varying ages and metallicities of the accreted dwarf galaxies forming the distant MW halo should give rise to variations in the ratios of the abundances of different stellar populations. Our mock haloes follow the star formation model outlined in Robertson et al. (2005) and Font et al. (2006), which produces a correlation between accretion time and metallicity since star formation ceases at accretion, and also produces a correlation between mass (or luminosity) and metallicity via the leaky-box implementation. Furthermore, GALAXIA uses a grid of stellar populations in age and metallicity (Sharma et al. 2011b) to compute the number of stellar tracers to generate. We expect that these assumptions will lead to each accreted structure in our mock haloes having a different ratio of the two tracers we have studied in this work, since the relative abundance of RRLe is higher at lower metallicity (Marconi et al. 2015). We have already seen that the RRLe and $\mathrm{M}$ giant abundances in our mock haloes depend differently on luminosity, as is shown in the upper-left panel of Fig. 8. Here we explore whether population ratios could be used to separate different accreted structures or to map out the accretion history.

First, we consider the variation in the number of $\mathrm{M}$ giants and RRLe among the different satellites in all our mock haloes. Fig. 14 shows that while as expected more luminous satellites (larger symbols) have more of both kinds of tracers than less luminous ones, we also see that in our model the very most luminous satellites tend to have more $\mathrm{M}$ giants than RRLe. The two panels of Fig. 14 illustrate how this trend arises from the star formation prescription in the model. On the left, we see the effect of truncating star formation (and hence chemical enrichment) at accretion: more recently, accreted satellites have more $M$ giants relative to RRLe. On the right, we see how this effect is transmitted to metallicity: the more metal-rich satellites do tend to have more $\mathrm{M}$ giants, but with more scatter than in accretion time. This indicates that our model should display variations in stellar populations analogous to those documented for the real halo by Bell et al. (2010), and that we should be able to disentangle different accretion events (at least for these mock haloes) by using ratios of stellar populations. If the model reflects the bulk properties of real chemical evolution in dwarf galaxies, we should also be able to use these ratios, combined with luminosity, 

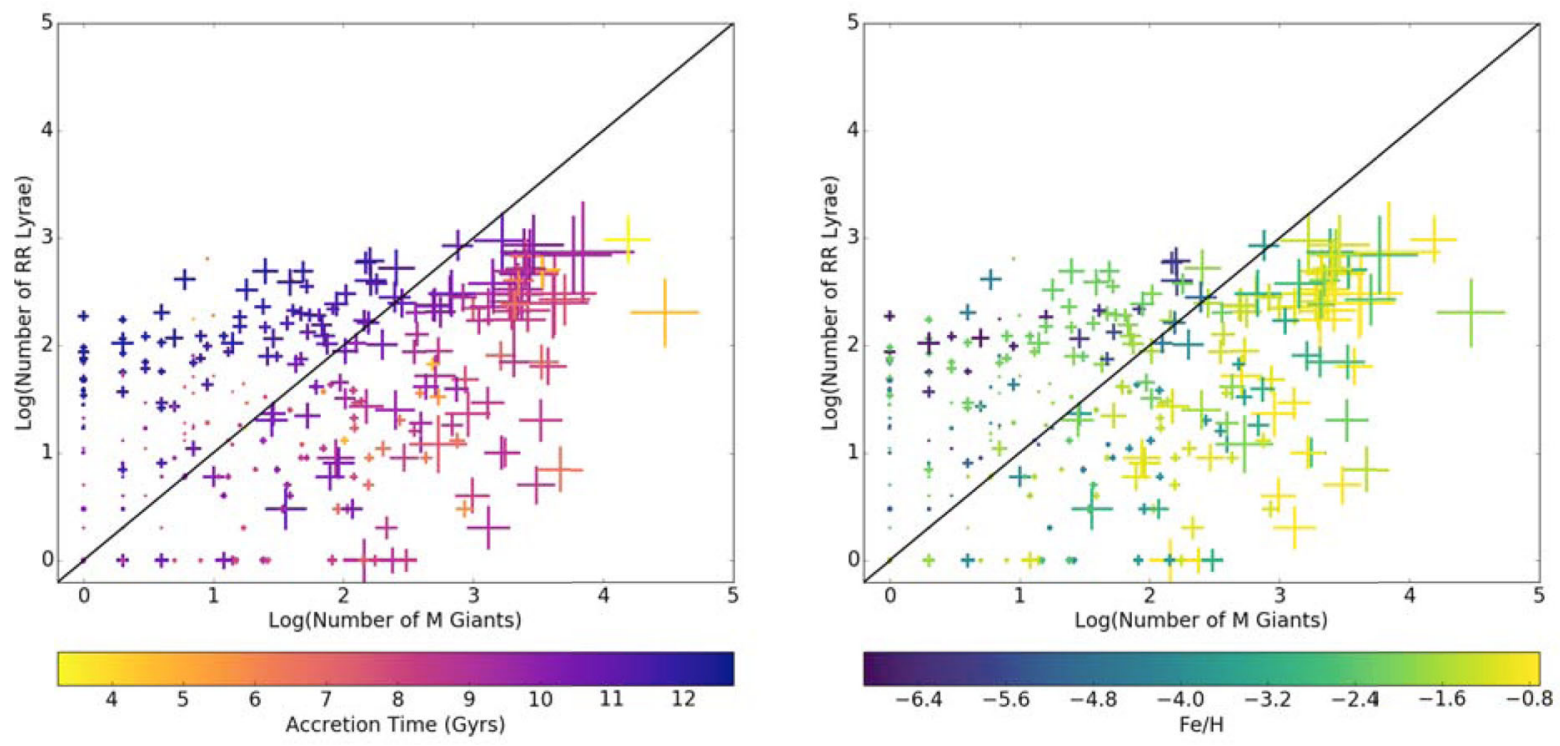

Figure 14. The distribution of the number of selected $M$ giants versus selected RRLe in unbound structures at the present day for each accreted building block. The black diagonal line represents a one-to-one ratio. The scale of each point is a function of total building block luminosity. The points are colour-coded by how long ago the building block was accreted in the left-hand panel and average $[\mathrm{Fe} / \mathrm{H}]$ in the right-hand panel.

to roughly date the different accretion events and reconstruct the Galactic accretion history.

Some differences between the two tracers are evident by eye when comparing the top-left and centre panels in Figs 11 and 10. The black colour-coded structure in Fig. 10 is very present and densely distributed in the $\mathrm{M}$ giant tracer, but looking at Fig. 11 it is barely noticeable in the RRL tracer. There is instead a smaller overdensity in the region from the small light blue structure. In the RRL maps of Fig. 11, we also see towards the right of the map a light green structure that provides a very visible overdensity in the density plot, but when looking at the $\mathrm{M}$ giant map, there are a few scattered green stars and no significant overdensity on the right. The differences in which tracers pick out which structures are mostly due to the range of metallicities of the building blocks, since only the low-metallicity building blocks contain any RRLe as shown in Fig. 14. That there are multiple different structures observable only in one or the other tracer is another argument for observing multiple tracers in order to uncover a more complete picture of the distant halo.

In the spirit of Bell et al. (2010), an all-sky view of the variations in ratio of M giants to RRLe for Halo 20 is shown in Fig. 15, where each point represents a star (either an M giant or RRL) colourcoded by the ratio of $\mathrm{M}$ giants to RRLe for its parent satellite. Only unbound structures are plotted. It is apparent that different structures have very different ratios of the two tracers, and that this can help distinguish overlapping structures. If some other way of determining membership was available, we could therefore use this information to reconstruct the accretion history. Based on the results of the previous sections, we tried linking overdensities in PM for RRLe and M giants in a mixed $2000 \mathrm{deg}^{2}$ field from one of our mock haloes, and looked at whether the resulting population ratios were close to those for the parent satellites. However, this fairly simplistic search method was not successful. We attribute this on one hand to the so-called curse of dimensionality: at these large distances, the structures are so sparsely sampled that in four dimensions (PMs, RV and distance) one rapidly ends up with zero or one star per bin even if each dimension is only divided into a few bins. On the other hand, because these structures are preferentially on very radial orbits, they tend to form shells rather than great-circle-like streams. This means that although these streams are still 'cold' in the sense that they are narrow distributions around a nearly one-dimensional path through six-dimensional phase space, the projection of that path into observed quantities is less well confined to a few observables than for great-circle streams, where it falls primarily along the sky coordinates with small gradients in velocity and distance. For a shell, the path falls mainly in the RV and distance space, but has a more complex shape in projection, and is generally double-valued in RV for a given distance. While there also exist templates for searching this space (Sanderson \& Helmi 2013), this points to a general need for a more sophisticated search method, so we defer this discussion to future work.

\section{CONCLUSIONS}

In this paper, we discuss prospects for observing and untangling the distant stellar halo in two tracers: $M$ giants and RRLe. We focused on the very distant stellar halo, beyond $100 \mathrm{kpc}$, where very few stars are presently detected. Using synthetic surveys of the 11 Bullock and Johnston mock stellar haloes, we found that the total number of tracers at distances beyond $100 \mathrm{kpc}$ varies by about an order of magnitude from halo to halo, with most of that variation due to the number of still-bound satellites. With these removed, the halo-to-halo variation is more like $0.5 \mathrm{dex}$. Excluding bound structures, our models predict roughly a few tens of thousands of $\mathrm{M}$ giants and a few thousand RRLe beyond $100 \mathrm{kpc}$, though these absolute numbers should be taken with caution since they depend strongly on our model's assumptions about the luminosities and stellar populations assigned to the building blocks.

Although the different mock haloes display wide variety, in general there are a few to half dozen large structures (besides the still-bound satellites) that are most prominent in a given halo and provide the best chance of identification. In quite a few cases, debris from the same accreted satellite is found on opposite sides of the sky with the RV signatures of shells (material piled up at apocentre), 


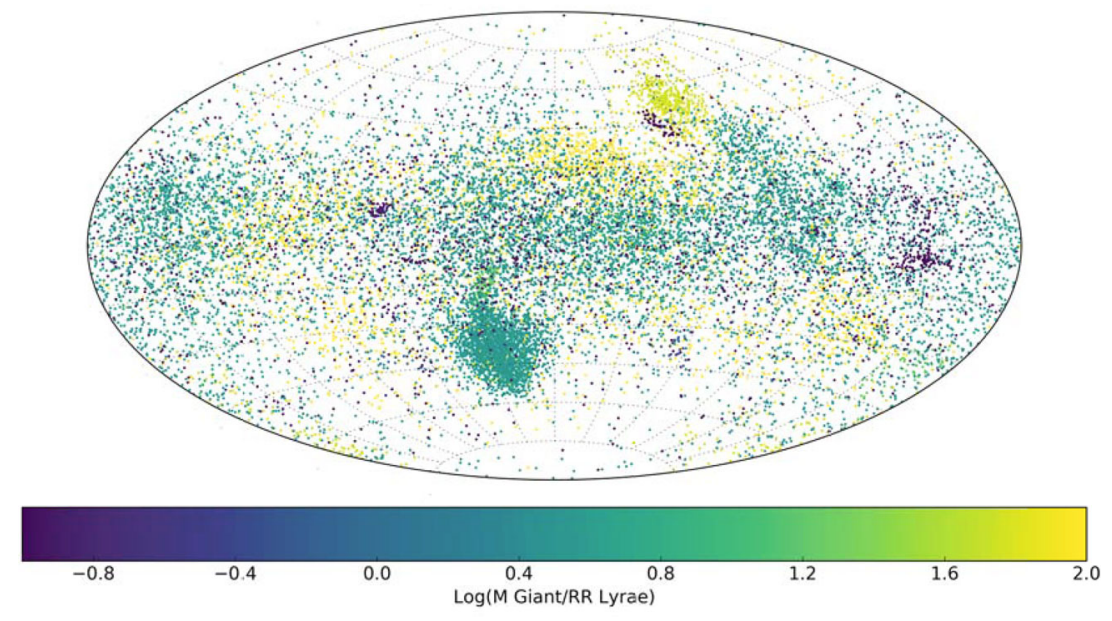

Figure 15. An all-sky plot of Halo 20. Each point represents either an RRLe within an error-convolved distance between 100 and $282 \mathrm{kpc}$ or a selected M giant. Points are colour-coded by the ratio of M giants to RRLe in its accretion satellite. Only unbound structures are plotted.

which is a result of the fairly radial orbits assigned to satellites by the model.

In studying the M giant tracers, we presumed photometric errors based on current data from UKIDSS, which had the effect of scattering stars into and out of the colour-colour boxes used to filter out foreground dwarf stars and background quasars. We found that these colour cuts, combined with a very loose PM selection, were effective at removing self-contamination by halo dwarf stars [Bochanski et al. (2014a) expect roughly 80 per cent contamination from foreground disc dwarf stars]. In the future, both infrared and visible photometry should improve substantially: the WFIRST HLS will cover roughly the same sky area as UKIDSS and have significantly better IR photometric quality. While UKIDSS has sub-0.1-mag photo errors to about 18th magnitude in the $J$ band, the WFIRST HLS will detect point sources to $5 \sigma$ down to 26.9 mag in the $J$ band (Spergel et al. 2013), although it will not cover the $K$ band. In the visible bands, LSST will see even deeper (down to $27.5 \mathrm{mag}$ in the $r$ band for $10 \mathrm{yr}$ co-adds). Thus, the technique of photometric selection should be promising for the next generation of surveys. The main challenge with so much data will be to improve the contamination by foreground disc stars, which may be possible with the improved PMs that such surveys will also provide.

We next considered a survey with a similar footprint size to UKIDSS, and found that a typical such footprint included stars from about $14 \pm 4$ different satellites. A typical survey of this size in our mock haloes is dominated by three to five of these satellites, each sampled by hundreds to thousands of $\mathrm{M}$ giants. These structures could be bound or unbound, but generally appear coherent on the sky. Doubling the survey size increases the number of component satellites by less than a factor of 2 , to a median of around 18 , and mainly has the effect of filling in the few most dominant structures.

We examined which components of position and velocity might offer the best chance of disentangling different accreted structures. At these distances, we found that PMs were more diagnostic than $\mathrm{RVs}$, which reflects the fact that most unbound structures at these distances are shells (and hence have a relatively wide range of $\mathrm{RVs}$ within each structure). Interestingly, the differences in PMs between accreted structures are of the order of $\sim 50-100 \mu \mathrm{as} \mathrm{yr}^{-1}$, comparable to the forecasted PM uncertainties for LSST in the optical (LSST Science Collaboration et al. 2009) and WFIRST in the infrared (Spergel et al. 2015). For M giants, we expect distance uncertainties to be too large (about 20 percent) to be useful for disentangling tracers, but for RRLe this coordinate should prove useful as well.

Finally, we considered the prospect of untangling accretion using ratios of $\mathrm{M}$ giants to RRLe. We found that this ratio does indeed vary substantially from one building block to another, with less luminous, older, more metal-poor satellites tending to have higher numbers of RRLe relative to $M$ giants. Given a way to confidently assign stars to structures, one could in principle use this dependence to map the accretion history of the outer halo, since the older/more metal-poor structures containing more RRLe also tend to have accreted earlier. However, our preliminary attempts to separate different structures by defining one- or two-dimensional ranges in PM space and searching the remaining coordinates for structure were not successful, indicating that a more sophisticated strategy that takes advantage of the full multidimensionality of the available data (Sharma \& Johnston 2009), and/or attempts to reduce the dimensionality to a few useful hybrid dimensions, will be needed to untangle the different accreted structures in the outer halo. Once this is done, it may be possible to then connect stars in structures on opposite sides of the sky using population ratios, which would provide valuable constraints on the MW dark matter distribution at large distances.

\section{ACKNOWLEDGEMENTS}

RES is supported by an NSF Astronomy and Astrophysics Postdoctoral Fellowship under grant AST-1400989. AS contributions were also partially supported by AST-1400989. KVJ and AS contributions were enabled by NSF grant AST-1312196. JJB acknowledges support from NSF CAREER grant AST-1151462.

\section{REFERENCES}

Abadi M. G., Navarro J. F., Steinmetz M., 2006, MNRAS, 365, 747

Bailin J., Bell E. F., Valluri M., Stinson G. S., Debattista V. P., Couchman H. M. P., Wadsley J., 2014, ApJ, 783, 95

Baker M., Willman B., 2015, AJ, 150, 160

Beaton R. L. et al., 2016, ApJ, 832, 210

Bell E. F. et al., 2008, ApJ, 680, 295

Bell E. F., Xue X. X., Rix H.-W., Ruhland C., Hogg D. W., 2010, AJ, 140 , 1850

Belokurov V. et al., 2006a, ApJ, 642, L137

Belokurov V. et al., 2006b, ApJ, 647, L111 
Bertelli G., Bressan A., Chiosi C., Fagotto F., Nasi E., 1994, A\&AS, 106

Bochanski J. J., Willman B., West A. A., Strader J., Chomiuk L., 2014a, AJ, 147,76

Bochanski J. J., Willman B., Caldwell N., Sanderson R., West A. A., Strader J., Brown W., 2014b, ApJ, 790, L5

Bullock J. S., Johnston K. V., 2005, ApJ, 635, 931

Bullock J. S., Kravtsov A. V., Weinberg D. H., 2001, ApJ, 548, 33

Clementini G., Gratton R., Bragaglia A., Carretta E., Di Fabrizio L., Maio M., 2003, AJ, 125, 1309

Cooper A. P. et al., 2010, MNRAS, 406, 744

De Lucia G., Helmi A., 2008, MNRAS, 391, 14

Deason A. J. et al., 2012, MNRAS, 425, 2840

Drlica-Wagner A. et al., 2015, ApJ, 813, 109

Ferguson A. M. N., Irwin M. J., Ibata R. A., Lewis G. F., Tanvir N. R., 2002, AJ, 124, 1452

Font A. S., Johnston K. V., Bullock J. S., Robertson B. E., 2006, ApJ, 638, 585

Font A. S., McCarthy I. G., Crain R. A., Theuns T., Schaye J., Wiersma R. P. C., Dalla Vecchia C., 2011, MNRAS, 416, 2802

Gilbert K. M., Font A. S., Johnston K. V., Guhathakurta P., 2009, ApJ, 701, 776

Helmi A., 2004, ApJ, 610, L97

Helmi A., Cooper A. P., White S. D. M., Cole S., Frenk C. S., Navarro J. F., 2011, ApJ, 733, L7

Ibata R. A. et al., 2014, ApJ, 780, 128

Ivezic Z. et al., 2008, Serb. Astron. J., 176, 1

Ivezić Ž., Beers T. C., Jurić M., 2012, ARA\&A, 50, 251

Johnston K. V., Law D. R., Majewski S. R., 2005, ApJ, 619, 800

Johnston K. V., Bullock J. S., Sharma S., Font A., Robertson B. E., Leitner S. N., 2008, ApJ, 689, 936

Koposov S. E., Rix H.-W., Hogg D. W., 2010, ApJ, 712, 260

Küpper A. H. W., Balbinot E., Bonaca A., Johnston K. V., Hogg D. W., Kroupa P., Santiago B. X., 2015, ApJ, 803, 80

Law D. R., Majewski S. R., 2010, ApJ, 714, 229

Law D. R., Johnston K. V., Majewski S. R., 2005, ApJ, 619, 807

Law D. R., Majewski S. R., Johnston K. V., 2009, ApJ, 703, L67

LSST Science Collaboration et al., 2009, preprint (arXiv:0912.0201)

Lux H., Read J. I., Lake G., Johnston K. V., 2012, MNRAS, 424, L16

Majewski S. R., Skrutskie M. F., Weinberg M. D., Ostheimer J. C., 2003, ApJ, 599, 1082
Marconi M. et al., 2015, ApJ, 808, 50

Marigo P., Girardi L., Bressan A., Groenewegen M. A. T., Silva L., Granato G. L., 2008, A\&A, 482, 883

Merrifield M. R., Kuijken K., 1998, MNRAS, 297, 1292

Newberg H. J. et al., 2002, ApJ, 569, 245

Newberg H. J., Willett B. A., Yanny B., Xu Y., 2010, ApJ, 711, 32

Pearson S., Küpper A. H. W., Johnston K. V., Price-Whelan A. M., 2015, ApJ, 799, 28

Pillepich A., Madau P., Mayer L., 2015, ApJ, 799, 184

Robertson B., Bullock J. S., Font A. S., Johnston K. V., Hernquist L., 2005, ApJ, 632, 872

Sanderson R. E., Helmi A., 2013, MNRAS, 435, 378

Schlaufman K. C. et al., 2009, ApJ, 703, 2177

Sesar B. et al., 2010, ApJ, 708, 717

Sesar B. et al., 2013, ApJ, 776, 26

Sesar B. et al., 2014, ApJ, 793, 135

Sharma S., Johnston K. V., 2009, ApJ, 703, 1061

Sharma S., Johnston K. V., Majewski S. R., Bullock J., Muñoz R. R., 2011a, ApJ, 728, 106

Sharma S., Bland-Hawthorn J., Johnston K. V., Binney J., 2011b, ApJ, 730,3

Spergel D. et al., 2013, preprint (arXiv:1305.5425)

Spergel D. et al., 2015, preprint (arXiv:1503.03757)

Tissera P. B., Scannapieco C., Beers T. C., Carollo D., 2013, MNRAS, 432, 3391

VanderPlas J. T., Ivezič Ž., 2015, ApJ, 812, 18

Vera-Ciro C., Helmi A., 2013, ApJ, 773, L4

Wechsler R. H., Bullock J. S., Primack J. R., Kravtsov A. V., Dekel A., 2002, ApJ, 568, 52

Wetzel A. R., Hopkins P. F., Kim J.-h., Faucher-Giguère C.-A., Kereš D., Quataert E., 2016, ApJ, 827, L23

Willett B. A., Newberg H. J., Zhang H., Yanny B., Beers T. C., 2009, ApJ, 697,207

Willman B. et al., 2005, ApJ, 626, L85

Xue X.-X. et al., 2011, ApJ, 738, 79

Zolotov A., Willman B., Brooks A. M., Governato F., Brook C. B., Hogg D. W., Quinn T., Stinson G., 2009, ApJ, 702, 1058

This paper has been typeset from a $\mathrm{T}_{\mathrm{E}} \mathrm{X} / \mathrm{LAT} \mathrm{T} \mathrm{X}$ file prepared by the author. 